OITS-663

NIKHEF-98-010

hep-ph/9812415

\title{
Next-To-Leading Order Cross Sections FOR TAGged REACTIONS
}

\author{
StÉPHANE KELLER \\ Institute of Theoretical Science \\ University of Oregon, Eugene, OR 97408, USA \\ ERIC LAENEN \\ NIKHEF Theory Group \\ P.O. Box 41882, 1009 DB Amsterdam, The Netherlands
}

\begin{abstract}
We extend the phase space slicing method of Giele, Glover and Kosower for performing next-to-leading order jet cross section calculations in two important ways: we show how to include fragmentation functions and how to include massive particles. These extensions allow the application of this method not just to jet cross sections but also to cross sections in which a particular final state particle, including a $D$ or $B$-meson, is tagged.
\end{abstract}




\section{Introduction}

The identification ("tagging") of one or more particular particles in the final state of reactions at colliders has been used succesfully in the past to enhance specific signals. A recent tremendous success was of course the top quark discovery [1] at the Tevatron, where $b$ quark tagging was used to suppress the background. It is expected that future analyses will increasingly rely on tagging various kinds of particles, witness the large effort spent on developing Cherenkov ("RICH") and micro-vertex detectors. Heavy ( $D$ and $B$ ) mesons in particular will certainly continue to play an essential part in this. It is imperative that the theoretical side of such analyses keep pace with these developments. A key ingredient of this theoretical effort must be the construction of fully exclusive Monte Carlo type programs, which include exact higher order QCD corrections and appropriate fragmentation functions that parametrize the transition of a parton to the particular hadron being tagged.

It is our goal to provide a systematic general-purpose method for constructing such Monte Carlo programs. In this paper we present the formalism. Numerical studies and applications will appear elsewhere [2].

Our method is an extended version of one already employed in the calculation of higher order jet cross sections, and is known as the "phase space slicing method" [3, 4. 4 .5]. One alternative method for calculating general jet cross sections is the "subtraction method" [6]. This method has also been applied to inclusive heavy quark cross sections [7]. Another, quite recent method is the "dipole method" [8], which can also be used for tagged reactions, and whose generalization to heavy quarks is in progress [9]. Yet another method, employing a small gauge boson mass, was used in [10]. The above methods differ in how they approximate the phase space and matrix elements in the neighborhood of divergent regions.

The particular version of the phase space slicing method we extend is given in the papers of Giele, Glover and Kosower in Refs. 国 and [5]. Their version minimizes computational effort in the calculation of jet cross sections by using the concepts of color ordering and crossing. The latter property, implemented in higher orders with the help of so-called crossing functions, allows the calculation of the matrix elements to be performed with all partons in the final state. We will employ both concepts here, and introduce the final state counterparts to the crossing functions, "tagging functions". In addition we compute the heavy quark contributions to the matrix elements, and to the crossing and tagging functions. The results presented in this paper have already partly been used in Ref. [11, and were briefly presented in [12].

The paper is organized as follows. In section 2 we review the basics of the phase space slicing method in the formalism of Giele, Glover and Kosower, and recall how it is used for computing general jet cross sections. In section 3 we extend their method to 
include fragmentation functions. The extension to heavy quarks is given in section 4 . We summarize and conclude in section 5.

\section{Phase Space Slicing Method for Jet Cross Sections}

In order to be self-contained and to establish notation used in later sections, we review in this section the phase space slicing (PSS) method, as given in Refs. [4,5], for calculating jet cross sections to next-to-leading order (NLO) in QCD. The method admits in principle an arbitrary number of jets in the final state, and is applicable to jet cross sections with either leptons or hadrons or both in the initial state. It allows for the study of any distribution of a given jet cross section, and for changes of jet-finding algorithms without analytical recalculation of matrix elements.

Consider the situation where at lowest order one has $n$ massless partons $\mathrm{p}$ in the final state produced by a vector boson $V$, i.e. the reaction:

$$
\left(l \overline{l^{\prime}} \rightarrow\right) V \rightarrow n \mathrm{p}
$$

$V$ itself can be the result of a lepton $(l)$ anti-lepton $\left(\bar{l}^{\prime}\right)$ collision. At this lowest order each parton is associated with its own jet, hence the matrix element for this reaction can be used to describe the process:

$$
\left(l \overline{l^{\prime}} \rightarrow\right) V \rightarrow n \text { jets }
$$

but also, after appropriate crossings and convolution with parton distribution functions, the reactions

$$
\begin{aligned}
h h^{\prime} & \rightarrow V\left(\rightarrow l \overline{l^{\prime}}\right)+(n-2) \text { jets } \\
l h & \rightarrow l^{\prime}+(n-1) \text { jets }
\end{aligned}
$$

where $h$ and $h^{\prime}$ are hadrons. One may also consider reactions without the vector boson $V$ in (3) and describe pure QCD reactions like:

$$
h h^{\prime} \rightarrow(n-2) \text { jets. }
$$

These reactions (35) represent the most prominent jet cross sections measured at colliders. When one performs the phase space integral over the final state parton momenta numerically, it is clear that at lowest order, where each parton forms a separate jet, one may impose any jet-defining cuts or acceptance cuts on the calculation. Although jet-finding algorithms can also be implemented easily, such a calculation cannot reproduce the details of the corresponding experimental cross section as it does not yield a prediction for the internal structure of a jet. 
It is the main purpose of the PSS method as described in Refs. [4.5] to maintain these features (i.e. the applicability of matrix elements to different processes, the easy numerical implementation of cuts and acceptance criteria, and the ability to change the jet algorithm without any analytical recalculations) at next-to-leading order.

The reasons for incorporating next-to-leading corrections in the modelling of jet cross sections are well-known:

- Reducing the normalization uncertainty via decreased sensitivity to changes in the renormalization scale and/or factorization scale.

- Testing the convergence of the QCD perturbation series for the observable under investigation.

- Modelling of inter-jet hadronic radiation.

- Modelling sensitivity to the jet algorithm.

The latter occurs because of the presence of the $n+1$ parton matrix element in the next-to-leading corrections. It is now possible for two partons to form a jet. However it is up to the particular jet algorithm to decide whether, and how, to merge a given pair of partons into a jet.

For definiteness we shall consider the process:

$$
V \rightarrow q \bar{q}+(n-2) g
$$

where we assume that the vector boson has been produced in an $e^{+} e^{-}$collision. For full details, we refer the reader to Ref. [4]. Later in this section we describe how to make the transition to the other reactions (3/5). That part of the discussion is a synopsis of Ref. [5].

At next-to-leading order, the contribution of the $n+1$-parton matrix element to the $n$-jet cross section in $e^{+} e^{-}$collisions involves integration over configurations where one parton is soft or two are collinear. In such situations divergences arise. They cancel against corresponding divergences in the virtual corrections to the $n$-parton contribution. The PSS method isolates these divergences by slicing the $n+1$-parton phase space into "hard" and "soft plus collinear" regions.

The hard region of phase space is essentially defined such that all of the $n+1$ partons are resolved: they are separated in phase space such that the invariant mass of any given two partons is larger than an arbitrary (small) theoretical cutoff $s_{\text {min }}$, $s_{i j}=\left(p_{i}+p_{j}\right)^{2}=2 p_{i} \cdot p_{j}>s_{\text {min }}$, where $p_{i}$ and $p_{j}$ represent the momenta of partons $i$ and $j$. In this region of phase space the $n+1$-parton cross section is finite and all phase space integrals may be done numerically. We may write this contribution as

$$
d \sigma^{R}\left(e^{+} e^{-} \rightarrow(n+1) \text { partons }\right)=\frac{1}{2^{2}} \frac{1}{2 Q^{2}}|M(1, \ldots, n+1)|^{2} d P^{R}(\mathcal{Q} ; 1, \ldots, n+1),
$$


where the spin averages and the flux factor have been shown explicitly, and $|M(. .)|^{2}$ is the $n+1$-parton LO matrix element squared and spin-summed. The resolved phase space in Eq. (7) is given by

$$
d P^{R}(\mathcal{Q} ; 1, \ldots, n+1)=\frac{1}{n_{g} ! \prod_{f} n_{q}^{f} ! \Pi_{f} n_{\bar{q}}^{f} !} \prod_{i j} \theta\left(s_{i j}-s_{\min }\right) d P(\mathcal{Q} ; 1, \ldots, n+1),
$$

where $Q$ stands for the sum of the momenta of the incoming electron and positron and $d P$ is the usual phase space measure in 4 dimensions:

$$
d P(Q ; 1, \ldots, n+1)=\left[\prod_{i=1}^{n+1} \frac{d^{3} \mathbf{p}_{i}}{(2 \pi)^{3} 2 E_{i}}\right](2 \pi)^{4} \delta\left(\mathcal{Q}-\sum_{i}^{n+1} p_{i}\right)
$$

An identical particle factor for gluons and (anti)quarks of the same flavor is included. The product over $\theta$ functions is defined such that all non-divergent, finite contributions are properly included in this resolved contribution. See below Eq. (17) for more detail.

In order to understand the behavior of the matrix elements in the soft and collinear region it is very useful to decompose the amplitude for producing $n+1$ partons in the final state using color-ordering [13]. To illustrate this, let us consider here for definiteness the amplitude for $n_{g}+1=n-1$ gluons radiating off the colored quark line that couples to the vector boson $V^{\mu}$,

$$
M(1, \ldots, n+1)_{c_{1} c_{2}}=V^{\mu} \hat{S}_{\mu}\left(K ; 1, \ldots, n_{g}+1 ; \bar{K}\right)_{c_{1} c_{2}}
$$

where $K, \bar{K}$ are the momenta of the final state quark and antiquark respectively and $c_{1}, c_{2}$ are their color indices. One may write

$\hat{S}_{\mu}\left(K ; 1, \ldots, n_{g}+1 ; \bar{K}\right)_{c_{1} c_{2}}=i e g^{n_{g}+1} \sum_{P\left(1 \ldots n_{g}+1\right)}\left(T^{a_{1}} \ldots T^{a_{n_{g}+1}}\right)_{c_{1} c_{2}} S_{\mu}\left(K ; 1, \ldots, n_{g}+1 ; \bar{K}\right)$.

Here $S_{\mu}\left(K ; 1, \ldots, n_{g}+1 ; \bar{K}\right)$ is a colorless subamplitude in which the $n_{g}+1$ gluons couple in a color ordered way to the quark line, and $e, g$ are the QED and QCD coupling constants respectively. The full amplitude is the sum over all permutations of these gluons.

A similar decomposition may be performed for the amplitude with four or more quarks in the final state. We shall not discuss such amplitudes in any detail. We merely note that such amplitudes are color-suppressed in relation to the gluonic ones, and can be straightforwardly included in the formalism (see Ref. [4] for details).

Using Eq. (11), the square of the full amplitude can be expanded in the number of colors $N$. One finds, after contraction with the vector boson $V$, and factoring out an overall factor $\left(N^{2}-1\right) / N$

$$
\left|V^{\mu} \hat{S}_{\mu}\right|^{2}=e^{2}\left(\frac{g^{2} N}{2}\right)^{n_{g}+1}\left(\frac{N^{2}-1}{N}\right)\left(A\left[n_{g}+1\right]+\frac{1}{N^{2}} B\left[n_{g}+1\right]+\ldots\right),
$$


where

$$
A\left[n_{g}+1\right]=\sum_{P\left(1 \ldots n_{g}+1\right)}\left|V^{\mu} S_{\mu}\left(K ; 1 \ldots n_{g}+1 ; \bar{K}\right)\right|^{2}
$$

There is no known general expression for the term $B\left[n_{g}+1\right]$, but for any given $n_{g}$ it may be computed straightforwardly [14]. Note also that Eq. (12) is a finite series. For example, with $n_{g}=1$ only the $A[2]$ and $B[2]$ terms are present, and $B[2]$ reads

$$
B[2]=-\left|V^{\mu}\left(S_{\mu}(K ; 1,2 ; \bar{K})+S_{\mu}(K ; 2,1 ; \bar{K})\right)\right|^{2} .
$$

Here the 3-gluon coupling cancels, and the gluons behave in fact like photons [14].

We now examine the complementary soft plus collinear region where one or two of the $s_{i j}$ are smaller than the theoretical cut-off, $s_{\min }$. In this region only $n$ of the $n+1$ partons are resolved. The divergences that occur in this region are regularized by working in $d=4-2 \epsilon$ dimensions. The advantage of the color-ordered decomposition of the matrix element is that an $n+1$-parton colorless ordered subamplitude factorizes into an $n$-parton amplitude and a 1-parton factor, for an appropiately small value of $s_{\text {min }}$. The $n+1$-parton phase space also factorizes into the $n$-parton phase space and a 1-parton factor. The integration of the 1-parton amplitude factor over the 1-parton phase space can easily be performed analytically.

After this integration the squared soft and collinear matrix elements, resp. $\left|V^{\mu} \hat{S}_{\mu}\right|_{S}^{2}$ and $\left|V^{\mu} \hat{S}_{\mu}\right|_{C}^{2}$ (they are given explicitly in Eqs. (25) and (38)), can be combined with the leading order (LO) and NLO virtual (V) n-parton contributions to form an effective $n$ resolved parton squared matrix element:

$$
|M(1, \ldots, n)|_{\mathrm{eff}}^{2}=|M(1, \ldots, n)|_{\mathrm{LO}+\mathrm{V}}^{2}+\left|V^{\mu} \hat{S}_{\mu}\right|_{S}^{2}+\left|V^{\mu} \hat{S}_{\mu}\right|_{C}^{2}
$$

After coupling constant renormalization $|M(1, \ldots, n)|_{\text {eff }}^{2}$ is finite, by virtue of the renormalizability of QCD [15] and the KLN theorem [16].

The resolved $n$ parton contribution is now given by:

$$
d \sigma^{R}\left(e^{+} e^{-} \rightarrow n \text { partons }\right)=\frac{1}{2^{2}} \frac{1}{2 Q^{2}}|M(1, \ldots, n)|_{\mathrm{eff}}^{2} d P^{R}(\mathcal{Q} ; 1, \ldots, n)
$$

Note that because $|M(1, \ldots, n)|_{\text {eff }}^{2}$ is finite, we may take the $n$-parton phase space measure here in 4 dimensions.

Combining Eqs. (16) and (7), we may now write the next-to-leading order $n$-jet cross section in $e^{+} e^{-}$collisions as

$$
d \sigma\left(e^{+} e^{-} \rightarrow n \text { jets }\right)=\Theta\left[d \sigma^{R}\left(e^{+} e^{-} \rightarrow n \text { partons }\right)+\int d \sigma^{R}\left(e^{+} e^{-} \rightarrow(n+1) \text { partons }\right)\right]
$$

Here $\Theta$ represents all the experimental effects, including the jet algorithm. The integral in the second term represents the projection of the phase space of the $n+1$ resolved 
partons onto the $n$ jets phase space. We can now be more explicit about the definition of the product over the $\theta$-functions in Eq. (8), which is implicit in the second term in Eq. (17). For each color order contributing to $|M(1, \ldots, n+1)|^{2}$, the product runs over no more than two invariants involving the same momentum, otherwise some finite contributions of order $s_{\min } / s_{\min }$ might not be properly included.

Eq. (17) now involves only finite quantities and may be used to construct a fully differential NLO Monte Carlo program for the production of $n$ jets in $e^{+} e^{-}$collisions. Note that both terms on the right hand side of Eq. (17) depend on $s_{\text {min }}$, but that no observable may depend on it, as long as $s_{\min }$ is taken small enough such that the approximations used in the soft and collinear region are valid. For a given process this must be checked numerically with the Monte Carlo program.

We now briefly describe the procedure to calculate the soft and collinear contributions.

\subsection{Soft Behavior of Matrix Element, Phase Space, and Cross Section}

Colorless ordered subamplitudes share the property of QED amplitudes that, in the limit where one of the external gauge bosons becomes soft, the amplitude factorizes into a lower order amplitude and an eikonal factor:

$$
\begin{aligned}
S_{\mu}\left(K ; 1, \ldots, n_{g}, s ; \bar{K}\right) & \rightarrow e_{\lambda}\left(n_{g} ; s ; \bar{K}\right) S_{\mu}\left(K ; 1, \ldots, n_{g} ; \bar{K}\right), \\
S_{\mu}\left(K ; 1, \ldots, m, s, m+1, \ldots, n_{g} ; \bar{K}\right) & \rightarrow e_{\lambda}(m ; s ; m+1) S_{\mu}\left(K ; 1, \ldots, n_{g} ; \bar{K}\right), \\
S_{\mu}\left(K ; s, 1, \ldots, n_{g} ; \bar{K}\right) & \rightarrow e_{\lambda}(K ; s ; 1) S_{\mu}\left(K ; 1, \ldots, n_{g} ; \bar{K}\right),
\end{aligned}
$$

where the eikonal factor is given by

$$
e_{\lambda}(a ; s ; b)=\left(\frac{\epsilon_{\lambda}(s) \cdot p_{a}}{p_{a} \cdot p_{s}}-\frac{\epsilon_{\lambda}(s) \cdot p_{b}}{p_{b} \cdot p_{s}}\right) .
$$

Here $\epsilon_{\lambda}^{\mu}(s)$ is the polarization vector of the soft gluon with momentum $s$ and helicity $\lambda$, and the momenta $p_{a}$ and $p_{b}$ are those of the two hard partons adjacent to the soft gluon in the specific color order considered. Summing over the helicities of the soft gluon one obtains for the square of the amplitude

$$
\begin{aligned}
\left|V^{\mu} \hat{S}_{\mu}\right|^{2} & \rightarrow e^{2}\left(\frac{g^{2} N}{2}\right)^{n_{g}}\left(\frac{N^{2}-1}{N}\right)\left[\sum_{P\left(1 \ldots n_{g}\right)} s_{F}\left(K ; 1, \ldots, n_{g} ; \bar{K}\right)\left|V^{\mu} S_{\mu}\left(K ; 1, \ldots, n_{g} ; \bar{K}\right)\right|^{2}\right. \\
& \left.+O\left(\frac{1}{N^{2}}\right)\right]
\end{aligned}
$$

where

$$
s_{F}\left(K ; 1, \ldots, n_{g} ; \bar{K}\right)=\left(\frac{g^{2} N}{2}\right)\left[\sum_{\lambda}\left|e_{\lambda}(K ; s ; 1)\right|^{2}+\ldots+\sum_{\lambda}\left|e_{\lambda}\left(n_{g} ; s ; \bar{K}\right)\right|^{2}\right] .
$$


In the soft limit the $d$-dimensional $(d=4-2 \epsilon) n+1$-parton phase space measure,

$$
d P^{d}(\mathcal{Q} ; 1, \ldots, n+1)=\left[\prod_{i=1}^{n+1} \frac{d^{d-1} \mathbf{p}_{i}}{(2 \pi)^{d-1} 2 E_{i}}\right](2 \pi)^{d} \delta\left(\mathcal{Q}-\sum_{i} p_{i}\right)
$$

also factorizes, up to terms of order $s_{\min } / \mathcal{Q}^{2}$, according to

$$
d P^{d}(\mathcal{Q} ; 1, \ldots, a, s, b, \ldots, n+1) \rightarrow d P^{d}(\mathcal{Q} ; 1, \ldots, n) d P_{\text {soft }}^{\epsilon}(a, s, b),
$$

where

$$
d P_{s o f t}^{\epsilon}(a, s, b)=\frac{(4 \pi)^{\epsilon}}{16 \pi^{2} \Gamma(1-\epsilon)} \frac{d s_{a s} d s_{b s}}{s_{a b}}\left[\frac{s_{a s} s_{b s}}{s_{a b}}\right]^{-\epsilon} \theta\left(s_{\min }-s_{a s}\right) \theta\left(s_{\min }-s_{b s}\right) .
$$

The $\theta$-functions define the soft region formally. After combining (20), (21) and (24),

and integrating over $s_{a s}$ and $s_{b s}$ the effective squared matrix element with $n_{g}$ resolved gluons and two resolved quarks is

$$
\begin{aligned}
\left|V^{\mu} \hat{S}_{\mu}\right|_{S}^{2} & =e^{2}\left(\frac{g^{2} N}{2}\right)^{n_{g}}\left(\frac{N^{2}-1}{N}\right)\left[\sum_{P\left(1 \ldots n_{g}\right)} S_{F}\left(K ; 1, \ldots, n_{g} ; \bar{K}\right)\left|V^{\mu} S_{\mu}\left(K ; 1, \ldots, n_{g} ; \bar{K}\right)\right|^{2}\right. \\
& \left.+O\left(\frac{1}{N^{2}}\right)\right]
\end{aligned}
$$

with

$$
S_{F}\left(K ; 1, \ldots, n_{g} ; \bar{K}\right)=\left(\frac{\alpha_{s} N}{2 \pi}\right) \frac{1}{\Gamma(1-\epsilon)}\left(\frac{4 \pi \mu^{2}}{s_{\min }}\right)^{\epsilon} \frac{1}{\epsilon^{2}}\left[\left(\frac{s_{K 1}}{s_{\min }}\right)^{\epsilon}+\ldots+\left(\frac{s_{n_{g}} \bar{K}}{s_{\min }}\right)^{\epsilon}\right] .
$$

Here $\mu$ is the dimension regularization scale, and $\alpha_{s}=\alpha_{s}(\mu)=g^{2} \mu^{-2 \epsilon} / 4 \pi$. We may interpret the dimensional regularization scale $\mu$ here already as the renormalization scale $\mu_{R}$, as coupling constant renormalization is implicitly assumed. Note that the identical particle factor in Eq. (8), $1 /\left(n_{g}+1\right)$ !, is changed to $1 / n_{g}$ ! in Eq. (23), thereby taking into account the $\left(n_{g}+1\right)$ possibilities of choosing a soft gluon. The contribution to $\left|V^{\mu} \hat{S}_{\mu}\right|_{S}^{2}$ at higher order in $1 / N$ can be calculated straightforwardly.

\subsection{Collinear Behavior of Matrix Element, Phase Space and Cross Section}

The matrix elements also develop a singularity when two of the partons, say $a$ and $b$, become collinear, i.e.

$$
\begin{array}{r}
p_{c}=p_{a}+p_{b} ; p_{a}=z p_{c} ; p_{b}=(1-z) p_{c} \\
s_{a b}=p_{c}^{2}=2 p_{a} \cdot p_{b}<s_{\min }
\end{array}
$$


where $s_{\text {min }}$ is again assumed to be small. In this case the full squared matrix element factorizes according to $\mathrm{H}$

$$
\left|V^{\mu} \hat{S}_{\mu}(1, \ldots, a, b, \ldots, n+1)\right|^{2} \rightarrow \hat{c}_{F}^{c \rightarrow a b}\left|V^{\mu} \hat{S}_{\mu}(1, \ldots, c, \ldots, n)\right|^{2} .
$$

It is useful to extract color factors via

$$
\hat{c}_{F}^{c \rightarrow a b}=\left(\frac{g^{2} N}{2}\right) f^{c \rightarrow a b} .
$$

Then we have

$$
f^{c \rightarrow a b}=\frac{P_{c \rightarrow a b}^{\epsilon}(z)}{s_{a b}} .
$$

The splitting functions $P_{c \rightarrow a b}^{\epsilon}$ read, in the conventional scheme (in which both external and internal particles are treated in $d=4-2 \epsilon$ dimensions)

$$
\begin{aligned}
P_{g \rightarrow g g}^{\epsilon}(z) & =2\left(\frac{1+z^{4}+(1-z)^{4}}{z(1-z)}\right), \\
P_{g \rightarrow q \bar{q}}^{\epsilon}(z) & =\frac{2}{N}\left(\frac{z^{2}+(1-z)^{2}-\epsilon}{1-\epsilon}\right), \\
P_{q \rightarrow q g}^{\epsilon}(z) & =2\left(1-\frac{1}{N^{2}}\right)\left(\frac{1+z^{2}-\epsilon(1-z)^{2}}{1-z}\right) .
\end{aligned}
$$

Notice that only when two adjacent partons in the subamplitude in a specific color order become collinear does one find a divergence. Here, the four quark $\left(+\left(n_{g}-1\right)\right.$ gluons $)$ amplitude, $\hat{T}_{\mu}$, must be considered: when a neighboring quark and antiquark form a flavor singlet and become collinear, then this amplitude also contributes to the $n$ parton amplitude. In analogy to Eq. (20), we have therefore in the collinear limit:

$$
\begin{aligned}
\left|V^{\mu} \hat{S}_{\mu}\right|^{2} & +\left|V^{\mu} \hat{T}_{\mu}\right|^{2} \rightarrow e^{2}\left(\frac{g^{2} N}{2}\right)^{n_{g}}\left(\frac{N^{2}-1}{N}\right) \\
& \times\left[\sum_{P\left(1 \ldots n_{g}\right)} c_{F}\left(K ; 1, \ldots, n_{g} ; \bar{K}\right)\left|V^{\mu} S_{\mu}\left(K ; 1, \ldots, n_{g} ; \bar{K}\right)\right|^{2}+O\left(\frac{1}{N^{2}}\right)\right]
\end{aligned}
$$

where

$$
c_{F}\left(K ; 1, \ldots, n_{g} ; \bar{K}\right)=\left(\frac{g^{2} N}{2}\right)\left[f^{K \rightarrow q g}+f^{1 \rightarrow g g}+\ldots+f^{\bar{K} \rightarrow g q}+n_{g} n_{f} f^{g \rightarrow q \bar{q}}\right] .
$$

The last term in this expression is due to $\hat{T}_{\mu}$. The factor $n_{g}$ in front of this last term arises because the identical particle factor in Eq. (8) for the four quarks contribution changes from $1 /\left(n_{g}-1\right)$ ! to $1 / n_{g}$ !. The factor $n_{f}$ results from summing over all (light) quark flavors. In the collinear region, defined in Eq. (27), the phase space measure also factorizes, up to terms of order $s_{\min } / Q^{2}$ as:

$$
d P^{d}(\mathcal{Q} ; 1, \ldots, n-1, a, b) \rightarrow d P^{d}(\mathcal{Q} ; 1, \ldots, n-1, c) d P_{\text {coll }}^{\epsilon}(a, b ; z),
$$

\footnotetext{
${ }^{1}$ In Ref. 狛 the notation $\hat{c}_{F}^{a b \rightarrow c}$ was used to indicate the splitting of parton $c$, instead of $\hat{c}_{F}^{c \rightarrow a b}$.
} 
where

$$
d P_{\text {coll }}^{\epsilon}(a, b ; z)=\frac{(4 \pi)^{\epsilon}}{16 \pi^{2} \Gamma(1-\epsilon)} d s_{a b} d z\left[s_{a b} z(1-z)\right]^{-\epsilon} \theta\left(s_{\min }-s_{a b}\right) .
$$

To obtain the collinear behavior of the cross section one needs to integrate the following expression:

$$
\begin{aligned}
\int\left(\frac{g^{2} N}{2}\right) f^{c \rightarrow a b} d P_{c o l l}^{\epsilon}(a, b ; z) & \equiv\left(\frac{\alpha_{s} N}{2 \pi}\right) \frac{\left(4 \pi \mu^{2}\right)^{\epsilon}}{\Gamma(1-\epsilon)} \\
& \times \int_{0}^{s_{\min }} d s_{a b} s_{a b}^{-1-\epsilon}\left[\frac{1}{4} \int_{z_{1}}^{1-z_{2}} d z[z(1-z)]^{-\epsilon} P_{c \rightarrow a b}^{\epsilon}(z)\right] \\
& =-\left(\frac{\alpha_{s} N}{2 \pi}\right) \frac{1}{\Gamma(1-\epsilon)}\left(\frac{4 \pi \mu^{2}}{s_{\min }}\right) \frac{1}{\epsilon} I_{c \rightarrow a b}\left(z_{1}, z_{2}\right)
\end{aligned}
$$

where the boundary values $z_{1}$ and $z_{2}$ prevent the integral from picking up contributions from the soft region:

$$
s_{a-1 a}>z_{1} s_{a-1 c}=s_{\min }, s_{b b+1}>\left(1-z_{2}\right) s_{c b+1}=s_{\min } .
$$

These boundaries clearly depend on the color order of the particular subamplitude $S_{\mu}$ under consideration. The collinear behavior of the effective squared matrix element with $n$ resolved partons, of which one is an unresolved collinear pair, is now given by

$$
\begin{aligned}
\left|V^{\mu} \hat{S}_{\mu}\right|_{C}^{2} & =e^{2}\left(\frac{g^{2} N}{2}\right)^{n_{g}}\left(\frac{N^{2}-1}{N}\right) \\
& \times\left[\sum_{P\left(1 \ldots n_{g}\right)} C_{F}\left(K ; 1, \ldots, n_{g} ; \bar{K}\right)\left|V^{\mu} S_{\mu}\left(K ; 1, \ldots, n_{g} ; \bar{K}\right)\right|^{2}+O\left(\frac{1}{N^{2}}\right)\right]
\end{aligned}
$$

where

$$
\begin{aligned}
C_{F}\left(K ; 1, \ldots, n_{g} ; \bar{K}\right) & =-\left(\frac{\alpha_{s} N}{2 \pi}\right) \frac{1}{\Gamma(1-\epsilon)}\left(\frac{4 \pi \mu^{2}}{s_{\min }}\right)^{\epsilon} \frac{1}{\epsilon}\left[I_{K \rightarrow q g}\left(0, \frac{s_{\min }}{s_{K 1}}\right)\right. \\
& \left.+I_{1 \rightarrow g g}\left(\frac{s_{\min }}{s_{K 1}}, \frac{s_{\min }}{s_{12}}\right)+\ldots+I_{\bar{K} \rightarrow g \bar{q}}\left(\frac{s_{\min }}{s_{n_{g} \bar{K}}}, 0\right)+n_{g} n_{f} I_{g \rightarrow q \bar{q}}(0,0) .\right]
\end{aligned}
$$

Note that when the boundary corresponds to a soft fermion, there is no singularity, so we can put the corresponding $z_{i}$ to zero. For completeness we list here the results for the $I$ functions, defined in Eq. (36), for massless partons [4, [5.

$$
\begin{gathered}
I_{g \rightarrow g g}\left(z_{1}, z_{2}\right)=\left(\frac{z_{1}^{-\epsilon}+z_{2}^{-\epsilon}-2}{\epsilon}\right)-\frac{11}{6}+\left(\frac{\pi^{2}}{3}-\frac{67}{18}\right) \epsilon+O\left(\epsilon^{2}\right), \\
I_{q \rightarrow q g}\left(z_{1}, z_{2}\right)=\left(1-\frac{1}{N^{2}}\right)\left[\left(\frac{z_{2}^{-\epsilon}-1}{\epsilon}\right)-\frac{3}{4}+\left(\frac{\pi^{2}}{6}-\frac{7}{4}\right) \epsilon\right]+O\left(\epsilon^{2}\right), \\
I_{g \rightarrow q \bar{q}}\left(z_{1}, z_{2}\right)=\frac{1}{N}\left[\frac{1}{3}+\frac{5 \epsilon}{9}\right]+O\left(\epsilon^{2}\right) .
\end{gathered}
$$


For $I_{q \rightarrow q g}\left(z_{1}, z_{2}\right)$ the $1 / N^{2}$ term in fact contributes to the $\mathcal{O}\left(1 / N^{2}\right)$ terms in Eq. (38). We use this definition of $I_{q \rightarrow q g}$ because it is more convenient for what follows. The remaining I's are trivially derived from these, via the relations

$$
I_{q \rightarrow g q}\left(z_{1}, z_{2}\right)=I_{q \rightarrow q g}\left(z_{2}, z_{1}\right) \quad, \quad I_{g \rightarrow \bar{q} q}\left(z_{1}, z_{2}\right)=I_{g \rightarrow q \bar{q}}\left(z_{2}, z_{1}\right)
$$

and

$$
I_{\bar{q} \rightarrow \bar{q} g}\left(z_{1}, z_{2}\right)=I_{q \rightarrow q g}\left(z_{1}, z_{2}\right) \quad, \quad I_{\bar{q} \rightarrow g \bar{q}}\left(z_{1}, z_{2}\right)=I_{q \rightarrow g q}\left(z_{1}, z_{2}\right) .
$$

Again, we refer to Ref. [4] for all details.

\subsection{Crossing}

In this subsection we review how to describe reactions involving initial state hadrons with the PSS method. The original derivation and all details can be found in Ref. [5].

The calculation of the NLO matrix elements in the previous section was done with all partons in the final state. To describe processes with initial state partons at NLO, one would like to generalize the crossing property of the LO matrix elements to NLO. In particular, one would like to use the already calculated effective matrix elements of Eq. (15), and do the crossing numerically, without having to redo the analytical calculation for the specific process under consideration. This is possible, via universal "crossing functions" [5]. The feasibility of thus extending crossing to NLO rests again upon the simultaneous factorization of phase space and matrix element for both initial and final state collinear radiation.

In general the NLO differential cross section for a process with initial hadrons $\mathcal{H}_{1}$ and $\mathcal{H}_{2}$ may be written as

$$
d \sigma_{\mathcal{H}_{1} \mathcal{H}_{2}}=\sum_{a, b} \int d x_{1} \int d x_{2} \mathcal{F}_{a}^{\mathcal{H}_{1}}\left(x_{1}\right) \mathcal{F}_{b}^{\mathcal{H}_{2}}\left(x_{2}\right) d \sigma_{a b}^{N L O}\left(x_{1}, x_{2}\right)
$$

where $a, b$ denote parton flavors and $x_{1}, x_{2}$ are parton momentum fractions. The symbols in this equation do not have quite the same meaning as they do in the standard factorization formula [17]. Here $\mathcal{F}_{a}^{\mathcal{H}}(x)$ is an "effective" parton distribution function and

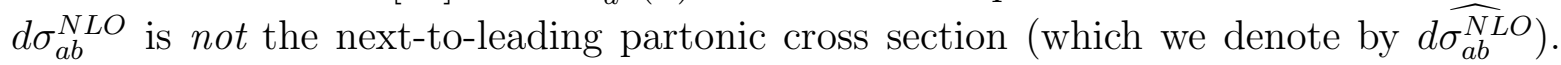
Rather, it consists of the finite effective all-partons-in-the-final-state matrix elements, in which partons $a$ and $b$ have simply been crossed to the initial state, i.e. in which their momenta $p_{a}$ and $p_{b}$ have been replaced by $-p_{a}$ and $-p_{b}$. The contributions that distinguish $d \sigma_{a b}^{N L O}$ from $d \widehat{\sigma_{a b}^{N L O}}$ are in fact included in $\mathcal{F}_{a}^{\mathcal{H}}(x)$ and are what distinguish the latter from the usual parton distribution functions $f_{a}^{\mathcal{H}}(x)$. Explicitly, we have

$$
d \sigma_{a b}^{N L O}=d \sigma_{a b}^{L O}+\alpha_{s} d\left(\delta \sigma_{a b}^{N L O}\right)+O\left(\alpha_{s}^{2}\right),
$$


with $\alpha_{s}=\alpha_{s}\left(\mu_{R}\right)$ from the crossed matrix elements, and

$$
\mathcal{F}_{a}^{\mathcal{H}}(x)=f_{a}^{\mathcal{H}}\left(x, \mu_{F}\right)+\alpha_{s} C_{a}^{\mathcal{H}}\left(x, \mu_{F}\right)+O\left(\alpha_{s}^{2}\right),
$$

where mass factorization has taken place at scale $\mu_{F} . f_{a}^{\mathcal{H}}\left(x, \mu_{F}\right)$ is a NLO parton distribution function and $C_{a}^{\mathcal{H}}\left(x, \mu_{F}\right)$ is a finite, process-independent crossing function.

There are two contributions that distinguish $d \sigma_{a b}^{N L O}$ from the full next-to-leading partonic cross section $d \widehat{\sigma_{a b}^{N L O}}$. First, when crossing a parton into the initial state we must correct for the possibility that this parton is in fact an unresolved collinear pair. Such "fusing" processes are not to be taken into account at leading twist and must hence be subtracted. Second, the contribution from the initial state collinear region must be included. Schematically, one has therefore, before mass factorization,

$$
C_{a}^{\mathcal{H}}(x) \sim \sum_{c, b}\left[\int_{x}^{1} \frac{d z}{z} f_{c}^{\mathcal{H}}\left(\frac{x}{z}\right) P_{c \rightarrow a b}(z)-f_{a}^{\mathcal{H}}(x) \int_{0}^{1} d z P_{a \rightarrow c b}(z)\right] \frac{s_{\min }^{-\epsilon}}{\epsilon},
$$

where the $f_{a}^{\mathcal{H}}$ are the bare distribution functions and the collinear singularities are still present. After mass factorization in a particular scheme, e.g. $\overline{\mathrm{MS}}$, one may write

$$
C_{a}^{\mathcal{H}, \overline{\mathrm{MS}}}\left(x, \mu_{F}\right)=\frac{N}{2 \pi}\left[A_{a}^{\mathcal{H}}\left(x, \mu_{F}\right) \ln \left(\frac{s_{\mathrm{min}}}{\mu_{F}^{2}}\right)+B_{a}^{\mathcal{H}, \overline{\mathrm{MS}}}\left(x, \mu_{F}\right)\right] .
$$

The functions $A$ and $B$ can be expressed as convolution integrals over the parton distribution functions and are listed in Ref. [5]. The $B$ functions are scheme dependent. The crossing functions, being process independent (universal), need to be calculated only once for any set of parton distribution functions (see [18, 19,20 for recent sets).

The full NLO differential cross section can now be written as:

$$
\begin{aligned}
d \sigma_{\mathcal{H}_{1} \mathcal{H}_{2}} & =\sum_{a, b} \int d x_{1} \int d x_{2} f_{a}^{\mathcal{H}_{1}}\left(x_{1}, \mu_{F}\right) f_{b}^{\mathcal{H}_{2}}\left(x_{2}, \mu_{F}\right) d \sigma_{a b}^{N L O}\left(x_{1}, x_{2}\right)+ \\
& +\alpha_{s}\left(\mu_{F}\right)\left(C_{a}^{\mathcal{H}_{1}}\left(x_{1}, \mu_{F}\right) f_{b}^{\mathcal{H}_{2}}\left(x_{2}, \mu_{F}\right)+f_{a}^{\mathcal{H}_{1}}\left(x_{1}, \mu_{F}\right) C_{b}^{\mathcal{H}_{2}}\left(x_{2}, \mu_{F}\right)\right) d \sigma_{a b}^{L O}\left(x_{1}, x_{2}\right) \\
& =\sum_{a, b} \int d x_{1} \int d x_{2} f_{a}^{\mathcal{H}_{1}}\left(x_{1}, \mu_{F}\right) f_{b}^{\mathcal{H}_{2}}\left(x_{2}, \mu_{F}\right) d \widehat{\sigma_{a b}^{N L O}}
\end{aligned}
$$

where the scheme dependence of the individual functions is suppressed.

We have reviewed quite extensively the PSS method for the calculation of general jet cross sections involving massless partons, and now proceed to the main task of this paper, which is to generalize the method to include fragmentation functions (in the next section), and heavy quarks (in section 4).

\section{Fragmentation and Tagging Functions}

The PSS method can be extended to describe not just jet cross sections, but also cross sections in which a particular particle is identified or "tagged" in the final state. 
Here that particle is assumed to be a hadron $\mathcal{H}$. Descriptions for such processes require the introduction of a fragmentation function $D_{h}^{\mathcal{H}}(z)$, representing the probability of a parton $h$ to fragment into $\mathcal{H}$, with $z$ the momentum fraction of $h$ carried by $\mathcal{H}$. In this section we show how to include fragmentation functions in the PSS method.

Let us consider the NLO cross section for the production of a hadron $\mathcal{H}$ and $(n-1)$ jets in $e^{+} e^{-}$collisions.

$$
d \sigma_{\mathcal{H}}=\sum_{h} d \sigma_{h}^{N L O} \mathcal{D}_{h}^{\mathcal{H}}(z) d z
$$

where $\mathcal{D}_{h}^{\mathcal{H}}$ is an effective next-to-leading order fragmentation function, in a similar sense that $\mathcal{F}_{a}^{\mathcal{H}}$ in Eq. (45) was an effective next-to-leading order parton distribution function. $d \sigma_{h}^{N L O}$ is in fact given by Eq. (17), with one of the resolved partons fixed to have flavor $h$. The sum is over all parton flavors $h$ in the intermediate state. The goal is again to use the results already obtained for the production of $n$ jets at NLO without need to modify the effective matrix element for the resolved $n$-parton contribution (Eq. (15)). We may expand

$$
\begin{gathered}
d \sigma_{h}^{N L O}=d \sigma_{h}^{L O}+\alpha_{s} d\left(\delta \sigma_{h}^{N L O}\right)+O\left(\alpha_{s}^{2}\right), \\
\mathcal{D}_{h}^{\mathcal{H}}(z)=D_{h}^{\mathcal{H}}(z)+\alpha_{s} T_{h}^{\mathcal{H}}(z)+O\left(\alpha_{s}^{2}\right),
\end{gathered}
$$

where the $T_{h}^{\mathcal{H}}$ are the final state equivalents of the crossing functions, described in the previous section. We call them "tagging functions".

Substitution of Eqs. (52) and (53) into (51) gives up to order $\alpha_{s}$

$$
d \sigma_{\mathcal{H}}=\sum_{h}\left[\left(d \sigma_{h}^{L O}+\alpha_{s} d\left(\delta \sigma_{h}^{N L O}\right)\right) D_{h}^{\mathcal{H}}(z)+\alpha_{s} d \sigma_{h}^{L O} T_{h}^{\mathcal{H}}(z)\right] d z
$$

What follows is in fact very similar to the derivation of the crossing functions in Ref. [5]. The tagging functions consist of those corrections that render Eq. (54) correct.

In the evaluation of the contribution in the collinear region in section 2 , the integration over the momentum fraction $z$ was performed. This collinear integration should however have been done in convolution with the fragmentation functions. Therefore, the integrated contribution must first be subtracted, and then the properly convoluted contributions should be added. For a specific color ordering, the integrated contribution is given by:

$$
\alpha_{s} T_{h, i n t}^{\mathcal{H}}(z)=\sum_{p, u} \int \hat{c}_{F}^{h \rightarrow p u}(y) d P_{\text {coll }}^{\epsilon}(p, u ; y) D_{h}^{\mathcal{H}}(z)
$$

so that

$$
T_{h, \text { int }}^{\mathcal{H}}(z)=-\left(\frac{N}{2 \pi}\right) \frac{1}{\Gamma(1-\epsilon)}\left(\frac{4 \pi \mu^{2}}{s_{\min }}\right)^{\epsilon} \frac{1}{\epsilon} \sum_{p, u} I_{h \rightarrow p u}\left(y_{1}, y_{2}\right) D_{h}^{\mathcal{H}}(z),
$$

where the sum is over all allowed parton flavors. The $I_{h \rightarrow p u}$ are given in Eqs. (40-44). Here $y_{1}$ and $y_{2}$ are the equivalent of the color-order dependent boundaries $z_{1}$ and $z_{2}$ in Eq. (37). The complete tagging functions $T_{h}^{\mathcal{H}}$ will be independent of the color ordering. 
Next, we need to add the collinear terms having the proper convolution with fragmentation functions. For each specific color ordering, the resulting coefficient is

$$
\alpha_{s} T_{h, \text { conv }}^{\mathcal{H}}(z) d z=\sum_{p, u} \int d P_{\text {coll }}^{\epsilon}(p, u ; y) \hat{c}_{F}^{h \rightarrow p u}(y) D_{p}^{\mathcal{H}}(x) \delta(z-x y) d x
$$

where $y=E_{p} / E_{h}$ and $x=E_{\mathcal{H}} / E_{p}$, so that $z=E_{H} / E_{h}$ is an energy fraction. Using the expressions of section 2 , we find

$$
T_{h, \text { conv }}^{\mathcal{H}}(z)=-\left(\frac{N}{2 \pi}\right) \frac{1}{\Gamma(1-\epsilon)}\left(\frac{4 \pi \mu^{2}}{s_{\min }}\right)^{\epsilon} \frac{1}{\epsilon} \sum_{p, u} \int_{z}^{1-y_{2}} \frac{d y}{y}[y(1-y)]^{-\epsilon} \frac{1}{4} P_{h \rightarrow p u}^{\epsilon}(y) D_{p}^{\mathcal{H}}(z / y) .
$$

Other definitions of $z$ are equivalent, e.g. using the plus components rather than the energies. We have

$$
T_{h, \text { conv }}^{\mathcal{H}}(z)=-\left(\frac{N}{2 \pi}\right) \frac{1}{\Gamma(1-\epsilon)}\left(\frac{4 \pi \mu^{2}}{s_{\min }}\right)^{\epsilon} \frac{1}{\epsilon} \sum_{p, u} \int_{z}^{1-y_{2}} \frac{d y}{y}[y(1-y)]^{-\epsilon} \frac{1}{4} P_{h \rightarrow p u}^{\epsilon}(y) D_{p}^{\mathcal{H}}(z / y) .
$$

This may be written as

$$
T_{h, \text { conv }}^{\mathcal{H}}(z)=-\left(\frac{N}{2 \pi}\right) \frac{1}{\Gamma(1-\epsilon)}\left(\frac{4 \pi \mu^{2}}{s_{\min }}\right)^{\epsilon} \frac{1}{\epsilon} \sum_{p} \int_{z}^{1} \frac{d y}{y} K_{h \rightarrow p}\left(y, y_{2}\right) D_{p}^{\mathcal{H}}(z / y),
$$

with

$$
K_{h \rightarrow p}\left(y, y_{2}\right)=\frac{1}{4} y^{-\epsilon}(1-y)^{-\epsilon} P_{h \rightarrow p u}^{\epsilon}(y) \theta\left(1-y-y_{2}\right) .
$$

The functions $K_{h \rightarrow p}\left(y, y_{2}\right)$ are equal to the functions $J_{p \rightarrow h}\left(y, y_{2}\right)$ in Ref. [5] in the crossing case, but multiplied by $y^{-\epsilon}$ and with $h$ and $p$ inverted. Using the plus prescripton

$$
F(z)_{+} \equiv \lim _{\beta \rightarrow 0}\left(\theta(1-z-\beta) F(z)-\delta(1-z-\beta) \int_{0}^{1-\beta} d y F(y)\right)
$$

we may expand the $K_{h \rightarrow p}\left(y, y_{2}\right)$ in order to extend the $y$-integral to 1 . For example, the right hand side in Eq. (60) contains terms that may be expanded as

$$
\begin{aligned}
\int_{z}^{1-y_{2}} d y \frac{g(y)}{(1-y)^{1+\epsilon}} & =\left(\frac{y_{2}^{-\epsilon}-1}{\epsilon}\right) g(1)+\int_{z}^{1} d y \frac{g(y)}{(1-y)_{+}} \\
& -\epsilon \int_{z}^{1} d y g(y)\left(\frac{\ln (1-y)}{1-y}\right)_{+}+\mathcal{O}\left(\epsilon^{2}\right) .
\end{aligned}
$$

Now combining the integrated and convoluted components leads to

$$
\begin{aligned}
T_{h}^{\mathcal{H}}(z) & =T_{h, \text { conv }}^{\mathcal{H}}(z)-T_{h, \text { int }}^{\mathcal{H}}(z) \\
& \equiv \sum_{p} \int_{z}^{1} \frac{d y}{y} Y_{h \rightarrow p}(y) D^{\mathcal{H}}{ }_{p}(z / y)
\end{aligned}
$$


The $Y_{h \rightarrow p}(y)$ are independent of the color ordering-dependent boundaries $y_{1}$ and $y_{2}$, so that $T_{h}^{\mathcal{H}}$ is indeed an overall factor multiplying the LO partonic cross section, as advertised in Eq. (54).

The functions $T_{h}^{\mathcal{H}}(z)$ in (64) still contain collinear singularities. These singularities are cancelled via mass factorization applied to the fragmentation functions, which in the present case amounts to writing

$$
D_{h}^{\mathcal{H}}(z)=D_{h}^{\mathcal{H}, \text { scheme }}\left(z, \mu_{D}\right)+\alpha_{s} \sum_{p} \int_{z}^{1} \frac{d y}{y} R_{h \rightarrow p}^{\text {scheme }}\left(y, \mu_{D}\right) D_{p}^{\mathcal{H}}\left(z / y, \mu_{D}\right)+O\left(\alpha_{s}^{2}\right),
$$

where $\mu_{D}$ is the final state factorization scale. The $R_{h \rightarrow p}\left(y, \mu_{D}\right)$ are counterterm functions, the finite terms of which determine the factorization scheme. In this section, where we are only dealing with massless partons, we choose the $\overline{\mathrm{MS}}$ counterterm functions $R_{h \rightarrow p}$, as in Ref. [5] for the crossing functions:

$$
\begin{gathered}
R_{q \rightarrow q}^{\overline{\mathrm{MS}}}\left(y, \mu_{D}\right)=\left(\frac{N}{2 \pi}\right)\left(\frac{4 \pi \mu^{2}}{\mu_{D}^{2}}\right)^{\epsilon} \frac{1}{\Gamma(1-\epsilon)} \frac{1}{\epsilon} \frac{1}{2}\left(1-\frac{1}{N^{2}}\right)\left[\left(\frac{1+y^{2}}{1-y}\right)\right]_{+}, \\
R_{q \rightarrow g}^{\overline{\mathrm{MS}}}\left(y, \mu_{D}\right)=\left(\frac{N}{2 \pi}\right)\left(\frac{4 \pi \mu^{2}}{\mu_{D}^{2}}\right)^{\epsilon} \frac{1}{\Gamma(1-\epsilon)} \frac{1}{\epsilon} \frac{1}{4} P_{q \rightarrow g q}^{\epsilon=0}(y), \\
R_{g \rightarrow q}^{\overline{\mathrm{MS}}}\left(y, \mu_{D}\right)=\left(\frac{N}{2 \pi}\right)\left(\frac{4 \pi \mu^{2}}{\mu_{D}^{2}}\right)^{\epsilon} \frac{1}{\Gamma(1-\epsilon)} \frac{1}{\epsilon} \frac{1}{4} P_{g \rightarrow q \bar{q}}^{\epsilon=0}(y) \\
R_{g \rightarrow g}^{\overline{\mathrm{MS}}}\left(y, \mu_{D}\right)=\left(\frac{N}{2 \pi}\right)\left(\frac{4 \pi \mu^{2}}{\mu_{D}^{2}}\right)^{\epsilon} \frac{1}{\Gamma(1-\epsilon)} \frac{1}{\epsilon}\left\{\frac{\left(11 N-2 n_{f}\right)}{6 N} \delta(1-y)\right. \\
\left.+2\left(\frac{z}{(1-z)_{+}}+\frac{(1-z)}{z}+z(1-z)\right)\right\}
\end{gathered}
$$

where $P_{c \rightarrow a b}^{\epsilon=0}$ can be inferred from the massless splitting functions in Eq. (31). The counterterm functions in Eqs. (66-69) cancel the $1 / \epsilon$ poles and the factor $(4 \pi)^{\epsilon} / \Gamma(1-\epsilon)$, present in $Y_{h \rightarrow p}$, and replace the dimensional regularization scale $\mu$ by the final state factorization scale $\mu_{D}$. Then we have

$$
T_{h}^{\mathcal{H}, \overline{\mathrm{MS}}}\left(z, \mu_{D}\right)=\sum_{p} \int_{z}^{1} \frac{d y}{y}\left(Y_{h \rightarrow p}(y)+R_{h \rightarrow p}^{\overline{\mathrm{MS}}}\left(y, \mu_{D}\right)\right) D_{p}^{\mathcal{H}}\left(z / y, \mu_{D}\right)
$$

Notice that the effective fragmentation function $\mathcal{D}$ is not dependent on the factorization scheme or scale $\mu_{D}$ :

$$
\mathcal{D}_{h}^{\mathcal{H}}(z)=D_{h}^{\mathcal{H}}\left(z, \mu_{D}\right)+\alpha_{s} T_{h}^{\mathcal{H}}\left(z, \mu_{D}\right)+O\left(\alpha_{s}^{2}\right) .
$$

The results of the calculation in Eq. (70) are summarized by

$$
T_{h}^{\mathcal{H}, \overline{\mathrm{MS}}}\left(z, \mu_{D}\right)=\frac{N}{2 \pi}\left[U_{h}^{\mathcal{H}}\left(z, \mu_{D}\right) \ln \left(\frac{s_{\mathrm{min}}}{\mu_{D}^{2}}\right)+V_{h}^{\mathcal{H}, \overline{\mathrm{MS}}}\left(z, \mu_{D}\right)\right],
$$


where

$$
U_{h}^{\mathcal{H}}\left(z, \mu_{D}\right)=\sum_{p} U_{h \rightarrow p}^{\mathcal{H}}\left(z, \mu_{D}\right), \quad V_{h}^{\mathcal{H}, \overline{\mathrm{MS}}}\left(z, \mu_{D}\right)=\sum_{p} V_{h \rightarrow p}^{\mathcal{H}, \overline{\mathrm{MS}}}\left(z, \mu_{D}\right)
$$

The functions $U_{h \rightarrow p}^{\mathcal{H}}$ are scheme independent and are given by:

$$
\begin{aligned}
& U_{g \rightarrow g}^{\mathcal{H}}\left(z, \mu_{D}\right)= \int_{z}^{1} \frac{d y}{y}\left[\frac{\left(11 N-2 n_{f}\right)}{6 N} \delta(1-y)+2\left(\frac{y}{(1-y)_{+}}+\frac{(1-y)}{y}\right.\right. \\
&+y(1-y))] D_{g}^{\mathcal{H}}\left(z / y, \mu_{D}\right), \\
& U_{q \rightarrow q}^{\mathcal{H}}\left(z, \mu_{D}\right)= \frac{1}{2}\left(1-\frac{1}{N^{2}}\right) \int_{z}^{1} \frac{d y}{y}\left[\frac{3}{2} \delta(1-y)+\frac{1+y^{2}}{(1-y)_{+}}\right] D_{q}^{\mathcal{H}}\left(z / y, \mu_{D}\right), \\
& U_{g \rightarrow q}^{\mathcal{H}}\left(z, \mu_{D}\right)=\frac{1}{4} \int_{z}^{1} \frac{d y}{y} P_{g \rightarrow q \bar{q}}^{\epsilon=0}(y) D_{q}^{\mathcal{H}}\left(z / y, \mu_{D}\right), \\
& U_{q \rightarrow g}^{\mathcal{H}}\left(z, \mu_{D}\right)=\frac{1}{4} \int_{z}^{1} \frac{d y}{y} P_{q \rightarrow g q}^{\epsilon=0}(y) D_{g}^{\mathcal{H}}\left(z / y, \mu_{D}\right),
\end{aligned}
$$

where the functions $P_{h \rightarrow p u}^{\epsilon=0}$ are obtained from Eq. (31). The scheme-dependent functions $V_{h \rightarrow p}^{\mathcal{H}, \overline{\mathrm{MS}}}$ can be similarly derived:

$$
\begin{gathered}
V_{g \rightarrow g}^{\mathcal{H}, \overline{\mathrm{MS}}}\left(z, \mu_{D}\right)=\int_{z}^{1} \frac{d y}{y}\left[2 \ln y\left(\frac{y}{(1-y)_{+}}+\frac{(1-y)}{y}+y(1-y)\right)\right. \\
+\left(\frac{\pi^{2}}{3}-\frac{67}{18}+\frac{5 n_{f}}{9 N}\right) \delta(1-y)+2 y\left(\frac{\ln (1-y)}{1-y}\right)_{+} \\
\left.+2\left(\frac{(1-y)}{y}+y(1-y)\right) \ln (1-y)\right] D_{g}^{\mathcal{H}}\left(z / y, \mu_{D}\right), \\
V_{q \rightarrow q}^{\mathcal{H}, \overline{\mathrm{MS}}}\left(z, \mu_{D}\right)=\frac{1}{2}\left(1-\frac{1}{N^{2}}\right) \int_{z}^{1} \frac{d y}{y}\left[\ln y \frac{1+y^{2}}{(1-y)_{+}}+\left(\frac{\pi^{2}}{3}-\frac{7}{2}\right) \delta(1-y)\right. \\
\left.+(1-y)+\left(1+y^{2}\right)\left(\frac{\ln (1-y)}{1-y}\right){ }_{+}\right] D_{q}^{\mathcal{H}}\left(z / y, \mu_{D}\right), \\
V_{g \rightarrow q}^{\mathcal{H}, \overline{\mathrm{MS}}}\left(z, \mu_{D}\right)=\frac{1}{4} \int_{z}^{1} \frac{d y}{y}\left[P_{g \rightarrow q \bar{q}}^{\epsilon=0}(y) \ln (y(1-y))-\hat{P}_{g \rightarrow q \bar{q}}^{\epsilon}(y)\right] D_{q}^{\mathcal{H}}\left(z / y, \mu_{D}\right), \\
V_{q \rightarrow g}^{\mathcal{H}, \overline{\mathrm{MS}}}\left(z, \mu_{D}\right)=\frac{1}{4} \int_{z}^{1} \frac{d y}{y}\left[P_{q \rightarrow g q}^{\epsilon=0}(y) \ln (y(1-y))-\hat{P}_{q \rightarrow g q}^{\epsilon}(y)\right] D_{g}^{\mathcal{H}}\left(z / y, \mu_{D}\right) .
\end{gathered}
$$

The functions $\hat{P}_{i \rightarrow j k}^{\epsilon}$ are defined by

$$
P_{i \rightarrow j k}^{\epsilon}=P_{i \rightarrow j k}^{\epsilon=0}+\epsilon \hat{P}_{i \rightarrow j k}^{\epsilon}+O\left(\epsilon^{2}\right),
$$


with the $P_{i \rightarrow j k}^{\epsilon}$ listed in Eq. (31). Notice the $\ln y$ terms generated by the extra factor $y^{-\epsilon}$ in the collinear phase space measure. We stress that the tagging functions are universal and need only to be calculated once for a given set of fragmentation functions (see [21] for recent sets).

We can combine the crossing functions and tagging functions in order to describe a NLO fully differential cross section for collisions of hadrons $\mathcal{H}_{1}$ and $\mathcal{H}_{2}$ in which hadron $\mathcal{H}$ is tagged in the final state:

$$
d \sigma_{\mathcal{H}_{1} \mathcal{H}_{2} \rightarrow \mathcal{H}+X}=\sum_{a, b, h} \int d x_{1} d x_{2} d z \mathcal{F}_{a}^{\mathcal{H} 1}\left(x_{1}\right) \mathcal{F}_{b}^{\mathcal{H} 2}\left(x_{2}\right) d \sigma_{a b h}^{N L O}\left(x_{1}, x_{2}, z\right) \mathcal{D}_{h}^{\mathcal{H}}(z)
$$

Substituting

$$
\begin{aligned}
d \sigma_{a b h}^{N L O}\left(x_{1}, x_{2}, z\right) & =d \sigma_{a b h}^{L O}\left(x_{1}, x_{2}, z\right)+\alpha_{s} d \delta \sigma_{a b h}^{N L O}\left(x_{1}, x_{2}, z\right)+O\left(\alpha_{s}^{2}\right), \\
\mathcal{F}_{a}^{\mathcal{H}}(x) & =f_{a}^{\mathcal{H}}\left(x, \mu_{F}\right)+\alpha_{s} C_{a}^{\mathcal{H}}\left(x, \mu_{F}\right)+O\left(\alpha_{s}^{2}\right), \\
\mathcal{D}_{h}^{\mathcal{H}}(z) & =D_{h}^{\mathcal{H}}\left(z, \mu_{D}\right)+\alpha_{s} T_{h}^{\mathcal{H}}\left(z, \mu_{D}\right)+O\left(\alpha_{s}^{2}\right),
\end{aligned}
$$

leads to

$$
\begin{aligned}
d \sigma_{\mathcal{H}_{1} \mathcal{H}_{2} \rightarrow \mathcal{H}+X}= & \sum_{a b h}\left[f_{a}^{\mathcal{H} 1}\left(x_{1}\right) f_{b}^{\mathcal{H} 2}\left(x_{2}\right)\left(d \sigma_{a b h}^{L O}\left(x_{1}, x_{2}, z\right)+\alpha_{s} d \delta \sigma_{a b h}^{N L O}\left(x_{1}, x_{2}, z\right)\right) D_{h}^{\mathcal{H}}(z)\right. \\
& +\alpha_{s} C_{a}^{\mathcal{H} 1}\left(x_{1}\right) f_{b}^{\mathcal{H} 2}\left(x_{2}\right) d \sigma_{a b h}^{L O}\left(x_{1}, x_{2}, z\right) D_{h}^{\mathcal{H}}(z) \\
& +\alpha_{s} f_{a}^{\mathcal{H} 1}\left(x_{1}\right) C_{b}^{\mathcal{H} 2}\left(x_{2}\right) d \sigma_{a b h}^{L O}\left(x_{1}, x_{2}, z\right) D_{h}^{\mathcal{H}}(z) \\
& \left.+\alpha_{s} f_{a}^{\mathcal{H} 1}\left(x_{1}\right) f_{b}^{\mathcal{H} 2}\left(x_{2}\right) d \sigma_{a b h}^{L O}\left(x_{1}, x_{2}, z\right) T_{h}^{\mathcal{H}}(z)+O\left(\alpha_{s}^{2}\right)\right] d x_{1} d x_{2} d z, \quad(87)
\end{aligned}
$$

where all the matrix elements in the partonic cross sections $\left(d \sigma_{a b h}^{L O}, d \sigma_{a b h}^{N L O}\right)$ are crossed versions of the ones with all resolved partons in the final state.

This completes the treatment of the massless fragmentation functions. We next extend the PSS formalism to include heavy quarks.

\section{Massive Particles}

A very important class of tags consists of heavy ( $\mathrm{D}$ or $\mathrm{B}$ ) mesons, indicating that a heavy quark was produced in the hard scattering. A description of such reactions with massless quarks is often not sufficiently accurate. In this section, we show how to extend the previous results to cases involving heavy quarks with an explicit mass. Throughout this section the mass is understood to be renormalized in the on-mass-shell scheme. 


\subsection{Soft Contribution}

We consider the reaction:

$$
\left(l \bar{l}^{\prime} \rightarrow\right) V \rightarrow Q_{1} \bar{Q}_{2}+\left(n_{g}+1\right) g
$$

where $V$ stands again for any vector boson (e.g. $\gamma, Z, W$, etc). Now $Q_{1}$ and $Q_{2}$ are heavy quarks with masses $m_{1}$ and $m_{2}$, respectively. Our results can be straightforwardly extended to the case with four massive quarks $V \rightarrow Q_{1} \bar{Q}_{2} Q_{3} \bar{Q}_{4}+\left(n_{g}-1\right) g$, or two heavy and two light ones, etc.

When gluon $s$ become soft, the colorless subamplitude $S_{\mu}$ again factorizes into an eikonal factor multiplying the ordered subamplitude with one less gluon. The square of the eikonal factor is, summed over the helicities of the soft gluon,

$$
f_{a b}(s)=\sum_{\lambda}\left|e_{\lambda}(a ; s ; b)\right|^{2}=\frac{4 s_{a b}}{s_{a s} s_{b s}}-\frac{4 m_{a}^{2}}{s_{a s}^{2}}-\frac{4 m_{b}^{2}}{s_{b s}^{2}},
$$

where we keep the definition of $s_{a s}$ as in section 2 :

$$
s_{a s} \equiv 2 p_{a} \cdot p_{s}
$$

and $m_{a}$ and $m_{b}$ are the masses of the neighboring particles, which can be $m_{1}, m_{2}$, or zero. To analyse the soft behavior of phase space, we only need to understand the three particle case, without loss of generality. The three particle phase space measure reads in $d=4-2 \epsilon$ dimensions

$$
d P^{d}(\mathcal{Q} ; a, b, s)=(2 \pi)^{3-2 d} \frac{d^{d-1} p_{a}}{2 E_{a}} \frac{d^{d-1} p_{b}}{2 E_{b}} \frac{d^{d-1} p_{s}}{2 E_{s}} \delta^{d}\left(\mathcal{Q}-p_{a}-p_{b}-p_{s}\right)
$$

The soft region is defined as in the massless case: $s_{a s}, s_{b s}<s_{\min }$, and $s_{\min }$ is assumed to be much smaller than $\mathcal{Q}^{2}$. This phase space measure again factorizes, up to terms of order $s_{\min } / Q^{2}$ :

$$
d P^{d}(\mathcal{Q} ; a, b, s) \rightarrow d P^{d}(\mathcal{Q} ; a, b) d P_{s o f t}^{\epsilon}(a, b, s) .
$$

The soft gluon measure

$$
d P_{\text {soft }}^{\epsilon}(a, b, s)=(2 \pi)^{1-d} \frac{d^{d-1} p_{s}}{2 E_{s}} \theta\left(s_{\min }-s_{a s}\right) \theta\left(s_{\min }-s_{b s}\right)
$$

may be written as

$$
\begin{aligned}
d P_{s o f t}^{\epsilon}(a, b, s)= & \frac{(4 \pi)^{\epsilon}}{16 \pi^{2}} \frac{\lambda^{\left(\epsilon-\frac{1}{2}\right)}}{\Gamma(1-\epsilon)}\left[s_{a s} s_{b s} s_{a b}-m_{b}^{2} s_{a b}^{2}-m_{a}^{2} s_{b s}^{2}\right]^{-\epsilon} \times \\
& d s_{a s} d s_{b s} \theta\left(s_{\min }-s_{a s}\right) \theta\left(s_{\min }-s_{b s}\right)
\end{aligned}
$$

with

$$
\lambda=s_{a b}^{2}-4 m_{a}^{2} m_{b}^{2}
$$


Note that if one takes $m_{a}$ and $m_{b}$ to zero, one recovers the massless result in Eq. (24). The factor between square brackets is in fact proportional to $\left(p_{s}^{\perp}\right)^{2}$, where $p_{s}^{\perp}$ is the component of $p_{s}$ in the direction perpendicular to $p_{a}$ in the center of mass frame of $a, b$, and $s$, and must hence be positive. This positivity condition leads to the kinematical constraint P

$$
\tau_{-} s_{b s}<s_{a s}<\tau_{+} s_{b s}
$$

or equivalently

$$
\sigma_{-} s_{a s}<s_{b s}<\sigma_{+} s_{b s}
$$

where

$$
\tau_{ \pm}=\frac{s_{a b}}{2 m_{b}^{2}} \pm \sqrt{\left(\frac{s_{a b}}{2 m_{b}^{2}}\right)^{2}-\frac{m_{a}^{2}}{m_{b}^{2}}}, \quad \sigma_{ \pm}=\frac{s_{a b}}{2 m_{a}^{2}} \pm \sqrt{\left(\frac{s_{a b}}{2 m_{a}^{2}}\right)^{2}-\frac{m_{b}^{2}}{m_{a}^{2}}} .
$$

Note that $\left(\tau_{ \pm}\right)^{-1}=\sigma_{\mp}$. For the rest of this discussion we assume $m_{a} \geq m_{b}$.

One can show that $\tau_{-} \leq(\geq) 1$ if $s_{a b} \geq(\leq) m_{a}^{2}+m_{b}^{2}$. Furthermore $\tau_{+}>1$. The complete integration region for $s_{a s}$ and $s_{b s}$ is conveniently divided into two regions. The first region is

$$
s_{b s} \in\left[0, \sigma_{-} s_{\min }\right], \quad s_{a s} \in\left[\tau_{-} s_{b s}, \tau_{+} s_{b s}\right],
$$

and the second region is

$$
\begin{array}{ll}
\text { 1) } \quad s_{a b} \geq m_{a}^{2}+m_{b}^{2}: & s_{b s} \in\left[\sigma_{-} s_{\min }, s_{\min }\right], \quad s_{a s} \in\left[\tau_{-} s_{b s}, s_{\min }\right], \\
\text { 2) } \quad s_{a b} \leq m_{a}^{2}+m_{b}^{2}: & s_{b s} \in\left[\sigma_{-} s_{\min }, \sigma_{+} s_{\min }\right], \quad s_{a s} \in\left[\tau_{-} s_{b s}, s_{\min }\right] .
\end{array}
$$

The threshold condition is $s_{a b} \geq 2 m_{a} m_{b}$. Note that $m_{b}=0$ implies $\sigma_{-}=0$, in which case the first region of integration does not contribute, and in the second region the lower limit of $s_{b s}$ reduces to zero.

Combining the soft part of the phase space and matrix element in this general massive case, we find

$$
S\left(m_{a}, m_{b}\right)=\int\left(\frac{g^{2} N}{2}\right) f_{a b}(s) d P_{s o f t}^{\epsilon}(a, b, s) .
$$

We distinguish between two cases in the rest of this section, because an extra divergence occurs when $m_{b}=0$. We find the following results after performing the integration in Eq. (101):

$m_{b}=0$ case

Denoting $m_{a} \equiv m$ we find

$$
S(m, 0)=\frac{\alpha_{s} N}{2 \pi} \frac{1}{\Gamma(1-\epsilon)}\left(\frac{4 \pi \mu^{2}}{s_{\min }}\right)^{\epsilon}\left(\frac{s_{a b}}{s_{\min }}\right)^{\epsilon} J(m, 0)
$$

\footnotetext{
${ }^{2}$ This result can also be derived from the condition that $|\cos (\theta)| \leq 1$, where $\theta$ is the angle between $a$ and $s$ in the center of mass frame of $a, b$, and $s$.
} 
where, corresponding to the two ranges in $(100)$

$$
\begin{gathered}
\text { 1) } s_{a b} \geq m^{2}: \quad J(m, 0)=\frac{1}{\epsilon^{2}}-\frac{1}{2 \epsilon^{2}}\left(\frac{s_{a b}}{m^{2}}\right)^{\epsilon}+\frac{1}{2 \epsilon}\left(\frac{s_{a b}}{m^{2}}\right)^{\epsilon}-\frac{1}{2} \zeta(2)+\frac{m^{2}}{s_{a b}} \\
\text { 2) } s_{a b} \leq m^{2}: \quad J(m, 0)=\left(\frac{s_{a b}}{m^{2}}\right)^{-\epsilon}\left(\frac{1}{2 \epsilon^{2}}+\frac{1}{2 \epsilon}-\frac{1}{2} \zeta(2)+1\right)
\end{gathered}
$$

Note that the strength of the $1 / \epsilon^{2}$ poles is the same in both cases.

$m_{b} \neq 0$ case

Here we find

$$
S\left(m_{a}, m_{b}\right)=\frac{\alpha_{s} N}{2 \pi} \frac{1}{\Gamma(1-\epsilon)}\left(\frac{4 \pi \mu^{2}}{s_{\min }}\right)^{\epsilon} \frac{\left(m_{b}^{2}\right)^{1-2 \epsilon}}{\lambda^{\frac{1}{2}}-\epsilon}\left(\frac{m_{b}^{2}}{s_{\min }}\right)^{\epsilon} J\left(m_{a}, m_{b}\right) .
$$

The contribution to $J$ coming from the first region of integration (99) has the following form:

$$
\begin{aligned}
J\left(m_{a}, m_{b}\right)= & -\frac{\tau_{+}^{2 \epsilon}}{2 \epsilon}\left(2\left(\tau_{-}-\tau_{+}\right)+\left(\tau_{-}+\tau_{+}\right) \ln \left(\frac{\tau_{+}}{\tau_{-}}\right)\right)-2\left(\tau_{+}-\tau_{-}\right) \ln \left(\tau_{+}-\tau_{-}\right) \\
& +\left(\tau_{+}-\tau_{-}\right)+\frac{\tau_{-}+\tau_{+}}{2} \ln \left(\frac{\tau_{+}}{\tau_{-}}\right)\left[1+2 \ln \left(\tau_{+}-\tau_{-}\right)\right] \\
& +\left(\frac{\tau_{-}+\tau_{+}}{2}\right)\left(\operatorname{Li}_{2}\left(1-\frac{\tau_{+}}{\tau_{-}}\right)-\operatorname{Li}_{2}\left(1-\frac{\tau_{-}}{\tau_{+}}\right)\right)
\end{aligned}
$$

The contribution from the second region of integration (100) is

$$
\begin{array}{ll}
\text { 1) } \quad s_{a b} \geq m_{a}^{2}+m_{b}^{2}: \quad J=1-\left(\tau_{-}+\tau_{+}\right)+\tau_{-} \tau_{+} \\
+\ln \left(\tau_{+}\right)\left(\tau_{-}-\tau_{+}-\left(\tau_{-}+\tau_{+}\right) \ln \left(\tau_{-}\right)\right)+\frac{1}{2}\left(\tau_{-}+\tau_{+}\right) \ln ^{2}\left(\tau_{+}\right) \\
\text {2) } \quad s_{a b} \leq m_{a}^{2}+m_{b}^{2}: \quad J=\left(\tau_{-}-\tau_{+}\right) \ln \left(\frac{\tau_{+}}{\tau_{-}}\right)+\frac{\left(\tau_{-}+\tau_{+}\right)}{2} \ln ^{2}\left(\frac{\tau_{+}}{\tau_{-}}\right) .
\end{array}
$$

We cannot take the limit $m_{b} \rightarrow 0$ in this expression, which reflects the extra singularity that leads to a double pole in Eq. (104). Note finally that if $m_{a}=m_{b}=m$ there is only the case $s_{a b} \geq 2 m^{2}$ (and $\sigma_{ \pm}=\tau_{ \pm}$), because the other case violates the threshold condition.

It is obvious how to modify the soft factor $S_{F}$ in Eq. (26) for reaction (88):

$$
\begin{aligned}
n_{g}>0 \quad: \quad S_{F}\left(Q_{1} ; 1, \ldots, n_{g} ; \overline{Q_{2}}\right)=\left(\frac{\alpha_{s} N}{2 \pi}\right) \frac{1}{\Gamma(1-\epsilon)}\left(\frac{4 \pi \mu^{2}}{s_{\min }}\right)^{\epsilon} \\
\times \quad\left[J\left(m_{1}, 0\right)\left(\frac{s_{K 1}}{s_{\min }}\right)^{\epsilon}+\frac{1}{\epsilon^{2}}\left(\frac{s_{12}}{s_{\min }}\right)^{\epsilon}+\ldots+\frac{1}{\epsilon^{2}}\left(\frac{s_{\left(n_{g}-1\right) 1}}{s_{\min }}\right)^{\epsilon}+J\left(0, m_{2}\right)\left(\frac{s_{n_{g} \bar{K}}}{s_{\min }}\right)^{\epsilon}\right] \\
n_{g}=0: \quad S_{F}\left(Q_{1} ; \overline{Q_{2}}\right)=\left(\frac{\alpha_{s}}{2 \pi}\right)\left(\frac{N^{2}-1}{N}\right) \frac{1}{\Gamma(1-\epsilon)}\left(\frac{4 \pi \mu^{2}}{s_{\min }}\right)^{\epsilon} \\
\times \quad\left[\frac{\left(m_{2}^{2}\right)^{1-2 \epsilon}}{\lambda^{1 / 2-\epsilon}}\left(\frac{m_{2}^{2}}{s_{\text {min }}}\right)^{\epsilon} J\left(m_{1}, m_{2}\right)\right] .
\end{aligned}
$$


Note the replacement of $N$ by $\left(N^{2}-1\right) / N$ in the case $n_{g}=0$.

There are no $1 / \epsilon$ singularities associated with the collinear region when one of the two collinear particles is a heavy quark. The mass of the quark screens the singularity. Therefore the above extension of the soft $K$-factor to the massive case is all that is needed to be able to apply the PSS method to processes involving heavy quarks. As far as UV renormalization is concerned in this situation, any scheme is of course allowed, but the Collins-Wilczek-Zee [22] scheme, in which the decoupling of the heavy quarks for small external momenta is manifest, is preferred. In this case only the light quarks contribute to the running of the QCD coupling.

The PSS method, extended as above to include soft radiation from heavy quarks, has in the recent past already been used in constructing NLO programs for reactions where heavy quarks are produced in leptonic collisions [23], and also in hadronic collisions using crossing functions [24]. We shall next extend it further to include collinear radiation.

\subsection{Collinear Behavior and Massless Limit}

In a scattering process involving a heavy quark in which the typical hard scale $Q$ (e.g. the $p_{T}$ of the heavy quark) is of the order of the heavy quark mass $m$, the results obtained thus far are adequate to describe the reaction. Singularities due to collinear configurations involving the heavy quark are screened by its mass, logarithms $\ln (\mathcal{Q} / m)$ are small, and the cross section is well-behaved. However if $\mathcal{Q} / m$ is large, the collinear region contributes large logarithms $\ln (\mathcal{Q} / m)$ that dominate the cross section at higher orders in perturbation theory. Moreover, they may spoil the convergence of the QCD perturbation series and one may wish to resum them. The $\ln (m)$ terms are the equivalent of the $1 / \epsilon$ poles that appear in the collinear region with massless partons in dimensional regularization and can be treated conceptually on equal footing. In what follows we describe how heavy quarks can be incorporated into the PSS formalism if $Q \gg m$.

The outline of this subsection is as follows. We first present results for the behavior of the cross section in the collinear region when the heavy quark is in the final state in the limit that $m / \mathcal{Q}$ and $m^{2} / s_{\min }$ are small. Then, we derive the heavy quark contribution to the tagging functions. We present results for two factorization schemes, a minimal substraction ("MS") and massive $\overline{\mathrm{MS}}($ " $\overline{\mathrm{MMS}}$ ") scheme, the latter defined such that the heavy quark fragmentation functions are the same as in the $\overline{\mathrm{MS}}$ massless case. We

show that the counterterm functions of the massive $\overline{\mathrm{MS}}$ scheme are in fact equal to the perturbative fragmentation functions of Ref. [25]. The fragmentation functions absorb in this way, through factorization, the $\ln (m)$ terms and resum the $\ln (\mathcal{Q} / m)$ terms through their evolution. We then present the equivalent results for the initial state.

A comprehensive treatment of heavy quark cross sections should be performed in the context of a variable flavor number scheme [26], which aims to describe the full region 
from $\mathcal{Q} \sim m$ to $Q \gg m$. This subject has recently been an active topic of investigation 27, 28, 29, 30,31,32. The results presented in this paper can be used to implement this scheme and we comment on this possibility at the end of this section.

\section{Final State}

We first derive the behavior of the cross section in the collinear limit when the typical hard scale $\mathcal{Q}$ is much larger than $m$. As in the massless case, we take all partons and massive quarks as outgoing. Because the mass serves as a regulator for the collinear divergences, we may work in four rather than in $d$ dimensions. We follow the structure of section 2.2. Let us denote the particles that become collinear by $A$ and $B$, and their parent particle by $C$,

$$
\begin{aligned}
p_{C} & =p_{A}+p_{B} ; \quad p_{A}=z p_{C} ; \quad p_{B}=(1-z) p_{C} \\
\delta_{A B} & =p_{C}^{2}-m_{C}^{2}=\left(p_{A}+p_{B}\right)^{2}-m_{C}^{2}<s_{\min } .
\end{aligned}
$$

$\delta_{A B}$ is the inverse propagator of $C$. The capital letters for the momenta remind us that two of the $A, B$ and $C$ particles are heavy quarks. We have defined $z$ in Eq. (111) as a 4-momentum fraction. Other definitions (such as an energy, or 3-momentum fraction) are equivalent in the collinear region when $m \ll \mathcal{Q}$. As usual we assume that $s_{\min }$ is small compared to $Q^{2}$. We furthermore neglect terms that give contributions of the order of $\mathrm{m}^{2} / \mathrm{s}_{\min }$ to the cross section.

As in the massless case, in the collinear region the matrix elements factorize:

$$
\left|V^{\mu} \hat{S}_{\mu}(1, \ldots, A, B, \ldots, n+1)\right|^{2} \rightarrow \hat{c}_{F}^{C \rightarrow A B}\left|V^{\mu} \hat{S}_{\mu}(1, \ldots, C, \ldots, n)\right|^{2}
$$

with

$$
\hat{c}_{F}^{C \rightarrow A B}=\left(\frac{g^{2} N}{2}\right) f^{C \rightarrow A B}
$$

and

$$
f^{C \rightarrow A B}=\frac{P_{C \rightarrow A B}^{m}\left(z, m, \delta_{A B}\right)}{\delta_{A B}} .
$$

The massive splitting functions $P_{C \rightarrow A B}^{m}$ contain a term involving the mass $m$ of the heavy quark $(Q)$ and $\delta_{A B}$ :

$$
\begin{gathered}
P_{Q \rightarrow Q g}^{m}\left(z, m, \delta_{Q g}\right)=2\left(1-\frac{1}{N^{2}}\right)\left[\left(\frac{1+z^{2}}{1-z}\right)-\frac{2 m^{2}}{\delta_{Q g}}\right], \\
P_{Q \rightarrow g Q}^{m}\left(z, m, \delta_{Q g}\right)=2\left(1-\frac{1}{N^{2}}\right)\left[\left(\frac{1+(1-z)^{2}}{z}\right)-\frac{2 m^{2}}{\delta_{Q g}}\right],
\end{gathered}
$$

where $\delta_{Q g}=2 p_{Q} \cdot p_{g}\left(\equiv s_{Q g}\right)$, and

$$
P_{g \rightarrow Q \bar{Q}}^{m}\left(z, m, \delta_{Q \bar{Q}}\right)=\frac{2}{N}\left[z^{2}+(1-z)^{2}+\frac{2 m^{2}}{\delta_{Q \bar{Q}}}\right]
$$


where $\delta_{Q \bar{Q}}=\left(p_{Q}+p_{\bar{Q}}\right)^{2}$. The terms containing the mass can be calculated by the methods used in [33.

The phase space also factorizes in the collinear region:

$$
d P^{4}(\mathcal{Q} ; 1, \ldots, n-1, A, B) \rightarrow d P^{4}(\mathcal{Q} ; 1, \ldots, n-1, C) d P_{\text {coll }}(A, B ; z),
$$

where

$$
d P_{\text {coll }}(A, B ; z)=\frac{1}{16 \pi^{2}} d z d \delta_{A B}
$$

The integrated collinear factor is then given by:

$$
\int\left(\frac{g^{2} N}{2}\right) f^{C \rightarrow A B} d P_{\text {coll }}(A, B ; z)=\left(\frac{\alpha_{s} N}{2 \pi}\right) I_{C \rightarrow A B}\left(z_{1}, z_{2}\right),
$$

where

$$
I_{C \rightarrow A B}\left(z_{1}, z_{2}\right)=\frac{1}{4} \int_{z_{1}}^{z_{2}} d z \int_{\delta_{\min }}^{s_{\min }} \frac{d \delta_{A B}}{\delta_{A B}} P_{C \rightarrow A B}^{m}\left(z, m, \delta_{A B}\right)
$$

$z_{1}$ and $z_{2}$ are defined as in the massless case, and are needed to avoid the soft region when $A$ or $B$ is a gluon. The kinematic limits $\delta_{\min }$ are different for each of the splittings and are given in Table 1. Note that although $m^{2}$ is small compared to $s_{\text {min }}$, we must

Table 1: The kinematic limits $\delta_{\min }$ for tagging functions.

\begin{tabular}{|c|c|}
\hline$C \rightarrow A B$ & $\delta_{\min }$ \\
\hline$Q \rightarrow Q g$ & $\frac{m^{2}(1-z)}{z}$ \\
\hline$Q \rightarrow g Q$ & $\frac{m^{2} z}{(1-z)}$ \\
\hline$g \rightarrow Q \bar{Q}$ & $\frac{m^{2}}{z(1-z)}$ \\
\hline
\end{tabular}

retain it in $\delta_{\text {min }}$, because it will give rise to $\ln \left(\mathrm{m}^{2} / \mathrm{s}_{\min }\right)$ contributions to the cross section that cannot be neglected. Other mass terms in the limits of integration that give rise to order $\left(\mathrm{m}^{2} / \mathrm{s}_{\min }\right)$ contributions have been neglected. We define at this point the $K_{C \rightarrow A}$ functions via:

$$
I_{C \rightarrow A B}\left(z_{1}, z_{2}\right)=\int_{z_{1}}^{1} d z K_{C \rightarrow A}\left(z, z_{2}\right) .
$$

When $z_{2}$ cannot be put to one (when $B$ is a gluon) its effect is absorbed in the integrand. With the plus prescription defined in Eq. (62), we obtain the following results:

$$
\begin{aligned}
K_{Q \rightarrow Q}\left(z, z_{2}\right) & =\frac{1}{2}\left(1-\frac{1}{N^{2}}\right)\left[\frac{1}{(1-z)_{+}}\left(\left(1+z^{2}\right) \ln \left(\frac{s_{\min }}{m^{2}} z\right)-2 z\right)\right. \\
& \left.-\left(1+z^{2}\right)\left(\frac{\ln (1-z)}{1-z}\right)_{+}-\delta(1-z)\left(2 \ln z_{2}\left(\ln \left(\frac{s_{\min }}{m^{2}}\right)-1\right)-\ln ^{2} z_{2}\right)\right]
\end{aligned}
$$




$$
\begin{gathered}
K_{Q \rightarrow g}(z, 0)=\frac{1}{2}\left(1-\frac{1}{N^{2}}\right)\left[\frac{1+(1-z)^{2}}{z} \ln \left(\frac{s_{\min }}{m^{2}} \frac{1-z}{z}\right)-\frac{2(1-z)}{z}\right] \\
K_{g \rightarrow Q}(z, 0)=\frac{1}{2 N}\left[\left(z^{2}+(1-z)^{2}\right) \ln \left(\frac{s_{\min }}{m^{2}} z(1-z)\right)+2 z(1-z)\right] .
\end{gathered}
$$

Consequently,

$$
\begin{aligned}
I_{g \rightarrow Q \bar{Q}}(0,0) & =\frac{1}{2 N} \int_{0}^{1} d z\left[\left(z^{2}+(1-z)^{2}\right) \ln \left(\frac{s_{\min }}{m^{2}} z(1-z)\right)+2 z(1-z)\right] \\
& =\frac{1}{2 N}\left(\frac{2}{3} \ln \left(\frac{s_{\min }}{m^{2}}\right)-\frac{10}{9}\right) \\
I_{Q \rightarrow Q g}\left(0, z_{2}\right) & =\frac{1}{2}\left(1-\frac{1}{N^{2}}\right) \int_{0}^{1-z_{2}} d z\left[\left(\frac{1+z^{2}}{1-z}\right) \ln \left(\frac{s_{\min }}{m^{2}} \frac{z}{1-z}\right)-\frac{2 z}{1-z}\right] \\
& =\frac{1}{2}\left(1-\frac{1}{N^{2}}\right)\left[\left(-2 \ln z_{2}-\frac{3}{2}\right) \ln \left(\frac{s_{\min }}{m^{2}}\right)+\ln ^{2} z_{2}+2 \ln z_{2}-\frac{\pi^{2}}{3}+\frac{3}{2}\right]
\end{aligned}
$$

and

$$
I_{Q \rightarrow g Q}\left(z_{1}, 0\right)=I_{Q \rightarrow Q g}\left(0, z_{1}\right)
$$

The massive $I_{C \rightarrow A B}$ functions can be used, as in the massless case, to calculate the collinear factor $C_{F}(\ldots)$ (see Eq. (39)) for each of the colorless subamplitudes. For sufficiently inclusive, infrared-safe observables, the $\ln \left(m^{2}\right)$ terms of the $I_{C \rightarrow A B}$ functions will cancel with corresponding terms of the virtual contribution, the same way the $1 / \epsilon$ terms cancel in the massless case. In these cases it is better to integrate the collinear region analytically as done here and perform the cancellations analytically. The numerical integration and cancellation, although always possible, is in practice difficult at large $\mathcal{Q}$.

We now turn to the problem of including the heavy quark into our formalism for fragmentation functions. For this we must add the heavy quark contribution to the tagging functions, where we assume that the transition of the heavy quark to a heavy hadron, at least for values of $\mathcal{Q}$ much larger than $m$, may be described by convoluting the partonic matrix element with a (non-perturbative) fragmentation function. In the normalization conventions of this section the heavy quark contribution to the tagging functions can be constructed via

$$
T_{h}^{\mathcal{H}}(z)=T_{H, \text { conv }}^{\mathcal{H}}(z)-T_{H, \text { int }}^{\mathcal{H}}(z) \equiv \sum_{p} \int_{z}^{1} \frac{d y}{y} Y_{H \rightarrow P}(y, m) D_{P}^{\mathcal{H}}(z / y),
$$

where

$$
T_{H, \text { conv }}^{\mathcal{H}}(z)=\left(\frac{N}{2 \pi}\right) \sum_{p} \int_{z}^{1} \frac{d y}{y} K_{H \rightarrow P}\left(y, y_{2}\right) D_{P}^{\mathcal{H}}\left(\frac{z}{y}\right)
$$


and

$$
T_{H, \text { int }}^{\mathcal{H}}(z)=\frac{N}{2 \pi} \sum_{P} I_{H \rightarrow P U}\left(y_{1}, y_{2}\right) D_{H}^{\mathcal{H}}(z) .
$$

$\mathcal{H}$ can now be a light or heavy hadron and $H, P$, and $U$ can be heavy quarks.

There are four possible contributions due to the heavy quark:

$$
\begin{aligned}
Y_{Q \rightarrow Q}(y, m) & =\left(\frac{N}{2 \pi}\right)\left(K_{Q \rightarrow Q}\left(y, y_{2}\right)-\delta(1-y) I_{Q \rightarrow Q g}\left(0, y_{2}\right)\right) \\
& =\left(\frac{N}{2 \pi}\right) \frac{1}{2}\left(1-\frac{1}{N^{2}}\right)\left[\left(\frac{1+y^{2}}{1-y}\right)_{+} \ln \left(\frac{s_{\text {min }}}{m^{2}}\right)+\frac{\left(1+y^{2}\right) \ln y}{(1-y)_{+}}\right. \\
& \left.-\frac{2 y}{(1-y)_{+}}-\left(1+y^{2}\right)\left(\frac{\ln (1-y)}{1-y}\right)_{+}+\left(\frac{\pi^{2}}{3}-\frac{3}{2}\right) \delta(1-y)\right], \\
Y_{g \rightarrow Q}(y, m) & =\left(\frac{N}{2 \pi}\right) K_{g \rightarrow Q}(y), \\
Y_{Q \rightarrow g}(y, m) & =\left(\frac{N}{2 \pi}\right) K_{Q \rightarrow g}(y),
\end{aligned}
$$

and

$$
Y_{g \rightarrow g}^{m}(y, m)=-\left(\frac{N}{2 \pi}\right) I_{g \rightarrow Q \bar{Q}}(0,0) \delta(1-y) .
$$

$Y_{g \rightarrow g}^{m}$ is the heavy quark contribution that must be added to the massless $Y_{g \rightarrow g}$. As in the massless case, all dependence on the boundary $y_{2}$ has cancelled. Eq. (54) can now also be applied to the massive fermion case. The heavy quark contribution to the tagging functions is independent of the hard process.

When $\ln (2 / m)$ becomes too large we need to these logarithms to all orders. This can be done by the evolution of the fragmentation functions, through factorization. The factorization procedure, as in Eq. (70), leads to scheme dependent $Y_{H \rightarrow P}$ functions:

$$
Y_{H \rightarrow P}(y, m) \rightarrow Y_{H \rightarrow P}^{\text {scheme }}\left(y, \mu_{D}\right)=Y_{H \rightarrow P}(y, m)+R_{H \rightarrow P}^{\text {scheme }}\left(y, m, \mu_{D}\right) .
$$

A minimal substraction, "MS", choice of the counterterm functions $R_{H \rightarrow P}$ is:

$$
\begin{gathered}
R_{Q \rightarrow Q}^{\mathrm{MS}}\left(y, m, \mu_{D}\right)=\left(\frac{N}{2 \pi}\right) \frac{1}{2}\left(1-\frac{1}{N^{2}}\right)\left[\left(\frac{1+y^{2}}{1-y}\right)\right]_{+} \ln \left(\frac{m^{2}}{\mu_{D}^{2}}\right), \\
R_{Q \rightarrow g}^{\mathrm{MS}}\left(y, m, \mu_{D}\right)=\left(\frac{N}{2 \pi}\right) \frac{1}{4} P_{q \rightarrow g q}^{\epsilon=0}(y) \ln \left(\frac{m^{2}}{\mu_{D}^{2}}\right) \\
R_{g \rightarrow Q}^{\mathrm{MS}}\left(y, m, \mu_{D}\right)=\left(\frac{N}{2 \pi}\right) \frac{1}{4} P_{g \rightarrow q \bar{q}}^{\epsilon=0}(y) \ln \left(\frac{m^{2}}{\mu_{D}^{2}}\right) \\
R_{g \rightarrow g}^{m, \mathrm{MS}}\left(y, m, \mu_{D}\right)=-\left(\frac{N}{2 \pi}\right) \frac{2}{6 N} \ln \left(\frac{m^{2}}{\mu_{D}^{2}}\right) \delta(1-y)
\end{gathered}
$$


where the $P_{c \rightarrow a b}^{\epsilon=0}$ can be inferred from the massless splitting functions in Eq. (31). $R_{g \rightarrow g}^{m}$ is the massive contribution that must be added to the massless $R_{g \rightarrow g}$. Notice that the heavy flavor is active in the evolution of the fragmentation function. The $Y_{H \rightarrow P}^{\mathrm{MS}}$ are now finite for $m \rightarrow 0$ at fixed $\mu_{D}$.

We can decompose the heavy quark contribution to the tagging functions as in Eq. (72) for the massless case, with:

$$
\begin{gathered}
U_{Q \rightarrow Q}^{\mathcal{H}}\left(z, \mu_{D}\right)=\frac{1}{2}\left(1-\frac{1}{N^{2}}\right) \int_{z}^{1} \frac{d y}{y}\left[\left(\frac{1+y^{2}}{1-y}\right)\right]_{+} D_{Q}^{\mathcal{H}}\left(z / y, \mu_{D}\right), \\
U_{g \rightarrow Q}^{\mathcal{H}}\left(z, \mu_{D}\right)=\frac{1}{4} \int_{z}^{1} \frac{d y}{y} P_{g \rightarrow q \bar{q}}^{\epsilon=0}(y) D_{Q}^{\mathcal{H}}\left(z / y, \mu_{D}\right), \\
U_{Q \rightarrow g}^{\mathcal{H}}\left(z, \mu_{D}\right)=\frac{1}{4} \int_{z}^{1} \frac{d y}{y} P_{q \rightarrow g q}^{\epsilon=0}(y) D_{g}^{\mathcal{H}}\left(z / y, \mu_{D}\right), \\
V_{Q \rightarrow Q}^{\mathcal{H}, \mathrm{MS}}\left(z, \mu_{D}\right)=\frac{1}{2}\left(1-\frac{1}{N^{2}}\right) \int_{z}^{1} \frac{d y}{y}\left[\ln y \frac{1+y^{2}}{(1-y)_{+}}+\left(\frac{\pi^{2}}{3}-\frac{3}{2}\right) \delta(1-y)\right. \\
\left.-\frac{2 y}{(\mathcal{H}}\left(z, \mu_{D}\right)=-\left(1+y_{g}{ }^{2}\right)\left(\frac{\ln (1-y)}{1-y}\right)_{+}\right] D_{Q}^{\mathcal{H}}\left(z / y, \mu_{D}\right), \\
V_{g \rightarrow Q}^{\mathcal{H}, \mathrm{MS}}\left(z, \mu_{D}\right)=\frac{1}{2 N} \int_{z}^{1} \frac{d y}{y}\left[\left(y^{2}+(1-y)^{2}\right) \ln (y(1-y))+2 y(1-y)\right] D_{Q}^{\mathcal{H}}\left(z / y, \mu_{D}\right),
\end{gathered}
$$

and

$$
\begin{gathered}
V_{Q \rightarrow g}^{\mathcal{H}, \mathrm{MS}}\left(z, \mu_{D}\right)=\frac{1}{2}\left(1-\frac{1}{N^{2}}\right) \int_{z}^{1} \frac{d y}{y}\left[\frac{1+(1-y)^{2}}{y} \ln \left(\frac{1-y}{y}\right)-\frac{2(1-y)}{y}\right] D_{g}^{\mathcal{H}}\left(z / y, \mu_{D}\right) . \\
V_{g \rightarrow g}^{m, \mathcal{H}, \mathrm{MS}}\left(z, \mu_{D}\right)=\frac{5}{9 N} D_{g}^{\mathcal{H}}\left(z, \mu_{D}\right)
\end{gathered}
$$

The $U^{\mathcal{H}}$ are scheme independent, and have the same functional dependence on their respective fragmentation functions as in the massless case. The contribution of the heavy quark to $U_{g \rightarrow g}$ and $V_{g \rightarrow g}$ increases the number of flavors $n_{f}$ in the massless expressions by one. After factorization the heavy quark contribution to the tagging functions is independent of $m$ and is therefore finite as $m$ goes to zero.

However, the tagging functions, although now $m$-independent, are not the same as those corresponding to the massless case of section 3. This implies that in the MS scheme the $m \rightarrow 0$ limit of the full partonic cross section, which can safely be taken, is not the same as if the calculation had been done with massless partons, and in the massless $\overline{\mathrm{MS}}$ scheme, from the outset. When $Q \gg m$, the most appropriate factorization scheme 
is therefore one where the subtracted tagging functions are the same as in the $\overline{\mathrm{MS}}$ massless scheme, up to small terms of order $m^{2} / \mathcal{Q}^{2}$. In other words, the counterterms in this massive $\overline{\mathrm{MS}}$ (" $\overline{\mathrm{MMS}}$ ") scheme must be such that $Y_{H \rightarrow P}^{\overline{\mathrm{MMS}}}\left(z, \mu_{D}\right)$ is equal to the corresponding massless function $Y_{h \rightarrow p}^{\overline{\mathrm{MS}}}\left(z, \mu_{D}\right)$. From this requirement we can straightforwardly infer from our earlier results what these $\overline{\mathrm{MMS}}$ counterterm functions must be, and we find

$$
\begin{gathered}
R_{Q \rightarrow Q}^{\overline{\mathrm{MMS}}}\left(y, m, \mu_{D}\right)=\left(\frac{N}{2 \pi}\right) \frac{1}{2}\left(1-\frac{1}{N^{2}}\right)\left[\left(\frac{1+y^{2}}{1-y}\right)_{+} \ln \left(\frac{m^{2}}{\mu_{D}^{2}}\right)+\frac{1+y^{2}}{(1-y)_{+}}\right. \\
\left.+2\left(\frac{\ln (1-y)}{1-y}\right)_{+}\left(1+y^{2}\right)-2 \delta(1-y)\right], \\
R_{Q \rightarrow g}^{\overline{\mathrm{MMS}}}\left(y, m, \mu_{D}\right)=\left(\frac{N}{2 \pi}\right) \frac{1}{4} P_{q \rightarrow g q}(y)\left(\ln \left(\frac{m^{2} y^{2}}{\mu_{D}^{2}}\right)+1\right), \\
R_{g \rightarrow Q}^{\overline{\mathrm{MMS}}}\left(y, m, \mu_{D}\right)=R_{g \rightarrow Q}^{\mathrm{MS}}\left(y, m, \mu_{D}\right) \\
R_{g \rightarrow g}^{m, \overline{\mathrm{MMS}}}\left(y, m, \mu_{D}\right)=R_{g \rightarrow g}^{m, \mathrm{MS}}\left(y, m, \mu_{D}\right)
\end{gathered}
$$

The choice of scheme has in turn implications for the fragmentation functions, which absorb these counterterm functions. They must be determined in other reactions in the same scheme. Notice from the above explicit expressions for the counterterms that the heavy quark contribution to the kernels for the evolution equations of the fragmentation functions are still those of the massless splitting functions.

We remark that when the contribution from the collinear region is calculated analytically in the $\overline{\mathrm{MMS}}$ scheme as described in this section, the massive counterterm functions are actually not needed. All that is needed to actually perform a calculation, besides the $\overline{\mathrm{MMS}}$ fragmentation functions, are the tagging functions, and in the $\overline{\mathrm{MMS}}$ scheme they are known by definition from the massless $\overline{\mathrm{MS}}$ case. However, when the contribution from the collinear region and the convolution with the $\overline{\mathrm{MMS}}$ fragmentation functions are computed numerically, then it is necessary to explicitly add the counterterm functions in Eqs. (150-153) to make the calculation consistent. In that case the equivalent tagging functions that should be used in Eq. (54) are given by:

$$
\sum_{P} \int_{z}^{1} \frac{d y}{y} R_{H \rightarrow P}^{\overline{\mathrm{MMS}}}\left(y, m, \mu_{D}\right) D_{P}^{\mathcal{H}}\left(z / y, \mu_{D}\right) .
$$

The original approach towards resumming the large logarithms in heavy quark production at high $Q^{2}$ is due to Mele and Nason in Ref. 25, and is called the heavy quark perturbative fragmentation functions (PFF) approach. Here the large logarithms $\ln (\mathcal{Q} / m)$ are summed at the partonic level. The resummation again occurs via evolution of the following initial distributions [25] at scale $\mu_{0}$ (of order $m$ ) to the large scale $\mathcal{Q}$

$$
D_{Q}\left(z, \mu_{0}, m\right)=\delta(1-z)+\frac{\alpha_{s}\left(\mu_{0}^{2}\right)}{2 \pi} d_{Q}^{(1)}\left(z, \mu_{0}, m\right)
$$


and

$$
D_{g}\left(z, \mu_{0}, m\right)=\frac{\alpha_{s}\left(\mu_{0}^{2}\right)}{2 \pi} d_{g}^{(1)}\left(z, \mu_{0}, m\right)
$$

In our formalism the $d_{H}^{(1)}$ are actually precisely given by the counterterm functions (multiplied by $-2 \pi)$ given in Eqs. (150-153), at $\mu_{D}=\mu_{0}$. The expressions in Eqs. (150) and (152) may be rewritten to obtain the same result as in Ref. [25]:

$$
\begin{gathered}
d_{Q}^{(1)}\left(z, \mu_{0}, m\right)=\frac{N^{2}-1}{2 N}\left[\left(\frac{1+z^{2}}{1-z}\right)\left(\ln \left(\frac{\mu_{0}^{2}}{m^{2}}\right)-1-2 \ln (1-z)\right)\right]_{+}, \\
d_{g}^{(1)}\left(z, \mu_{0}, m\right)=\left[z^{2}+(1-z)^{2}\right] \ln \left(\frac{\mu_{0}^{2}}{m^{2}}\right) .
\end{gathered}
$$

The PFF approach has been used to obtain resummed transverse momentum spectra for a variety of reactions [34, 35.

\section{Initial State}

In this section we present the results needed to include processes with heavy quarks in the initial state within the PSS formalism, when $Q \gg m$. As in the massless case we assume that the calculation of the squared matrix element was done with all partons, including the heavy quarks, in the final state. The heavy quark contribution from the collinear regions is computed analytically, as just described, under the assumption that $\mathcal{Q}$ is large. We now calculate the heavy quark contribution to the crossing functions. We follow the methods and notation of Ref. [5], as outlined in section 2.3. Recall that we must add the contributions from the initial state collinear region and subtract the contributions from the final state collinear region that are part of the analytical calculation but that do not contribute when the unresolved collinear pairs in the colorless amplitudes are crossed. As in the final state case, we neglect terms of order $\mathrm{m}^{2} / \mathrm{s}_{\min }$.

The process under consideration may be generically represented as

$$
P+a \rightarrow U+M
$$

where $P$ and $a$ are the initial particles of the hard subprocess and $M$ can decay into any number of particles. Particle $U$ is radiated collinearly off particle $P$ (and is not observed):

$$
P \rightarrow H+U
$$

whereas $H$ undergoes a hard scattering with $a$ to produce the final state labeled $M$. Here, up to two of $P, H$, and $U$ may be heavy quarks, hence the upper case notation. The momentum fraction $z$ is again defined by: $p_{H}=z p_{P}, p_{U}=(1-z) p_{P}$, see Eq. (111) and the comments below it. The inverse propagator of the off-shell particle $H$ is given 
by: $\delta_{P U}=\left(p_{P}-p_{U}\right)^{2}-m_{H}^{2}$. The collinear region is defined by $\left|\delta_{P U}\right|<s_{\min }$. In this limit the phase space and the flux factor factorize in the following way:

$$
\frac{1}{2 s_{P a}} d P(P+a \rightarrow U+M) \rightarrow d P_{\text {coll }}(P, U) \times \frac{1}{2 s_{H a}} d P(H+a \rightarrow M) .
$$

Note that $s_{\mathrm{Ha}}=z s_{\mathrm{Pa}}$, where the $z$ factor is included in $d P_{\text {coll }}(P, U)$. In our notation [ $s_{P a}=2 p_{a} \cdot p_{P}=\left(p_{a}+p_{P}\right)^{2}-m_{P}^{2}=\lambda^{1 / 2}\left(\left(p_{a}+p_{P}\right)^{2}, m_{P}^{2}, 0\right)$, where we have assumed that $m_{a}=0$.

The collinear phase space measure is given by:

$$
d P_{\text {coll }}(P, U)=\frac{1}{16 \pi^{2}} z d z d\left|\delta_{P U}\right|
$$

The full squared matrix element factorizes in the collinear region as described by Eq. (28) in the massless case. The $\hat{c}_{F}^{P \rightarrow H U}$ are now given by:

$$
\hat{c}_{F}^{P \rightarrow H U}=\left(\frac{g^{2} N}{2}\right) \frac{1}{z} \frac{P_{P \rightarrow H U}^{m}\left(z, m, \frac{\left|\delta_{P U}\right|}{z}\right)}{\left|\delta_{P U}\right|} .
$$

This result can be obtained by appropriate crossing of the final state $\hat{c}_{F}$-factor in Eq. (113). The massive splitting functions are given in Eqs. (115 117). The result for $\hat{c}_{F}^{g \rightarrow Q Q}$ in Eq. (163) is also given in Ref. 29.].

The initial state component of the crossing functions is given by

$$
\begin{aligned}
\alpha_{s} C_{H, \text { init }}^{\mathcal{H}}(x) & =\int \sum_{P, U} f_{P}^{\mathcal{H}}(y) \hat{c}_{F}^{P \rightarrow H U} d P_{\text {coll }}(P, U) \delta(x-z y) d y d z, \\
C_{H, \text { init }}^{\mathcal{H}}(x) & =\left(\frac{N}{2 \pi}\right) \sum_{P} \frac{1}{4} \int_{x}^{1-z_{2}} \frac{d z}{z} \int_{\delta_{\min }}^{s_{\min }} \frac{d\left|\delta_{P U}\right|}{\left|\delta_{P U}\right|} f_{P}^{\mathcal{H}}(x / z) P_{P \rightarrow H U}^{m}\left(z, m, \frac{\left|\delta_{P U}\right|}{z}\right), \\
& =\left(\frac{N}{2 \pi}\right) \sum_{P} \int_{x}^{1} \frac{d z}{z} f_{P}^{\mathcal{H}}(x / z) J_{P \rightarrow H}\left(z, z_{2}\right) .
\end{aligned}
$$

Here $\mathcal{H}$ is an initial state hadron, $f_{P}^{\mathcal{H}}(x)$ is the unrenormalized distribution function of $P$ in $\mathcal{H} . z_{2}$ is introduced as before to avoid the soft singularity when $U$ is a gluon. The kinematic limits $\delta_{\min }$ are given in Table 2 .

Using Eq. (62), we obtain

$$
\begin{aligned}
J_{Q \rightarrow Q}\left(z, z_{2}\right) & =\frac{1}{2}\left(1-\frac{1}{N^{2}}\right)\left[\frac{1}{(1-z)_{+}}\left(\left(1+z^{2}\right) \ln \left(\frac{s_{\min }}{m^{2}}\right)-2 z\right)-\left(1+z^{2}\right)\left(\frac{\ln (1-z)}{1-z}\right)_{+}\right. \\
& \left.-\delta(1-z)\left(2 \ln z_{2}\left(\ln \left(\frac{s_{\min }}{m^{2}}\right)-1\right)-\ln ^{2} z_{2}\right)\right], \\
J_{Q \rightarrow g}(z, 0) & =\frac{1}{2}\left(1-\frac{1}{N^{2}}\right)\left[\frac{1+(1-z)^{2}}{z} \ln \left(\frac{s_{\min }}{m^{2}} \frac{1-z}{z^{2}}\right)-\frac{2(1-z)}{z}\right], \\
J_{g \rightarrow Q}(z, 0) & =\frac{1}{2 N}\left[\left(z^{2}+(1-z)^{2}\right) \ln \left(\frac{s_{\min }}{m^{2}}(1-z)\right)+2 z(1-z)\right] . \\
\hline{ }^{3} \lambda(x, y, z)= & x^{2}+y^{2}+z^{2}-2 x y-2 x z-2 y z .
\end{aligned}
$$


Table 2: The kinematic limits $\delta_{\min }$ for the crossing functions.

\begin{tabular}{|c|c|}
\hline$p \rightarrow h u$ & $\delta_{\min }$ \\
\hline$Q \rightarrow Q g$ & $m^{2}(1-z)$ \\
\hline$Q \rightarrow g Q$ & $\frac{m^{2} z^{2}}{(1-z)}$ \\
\hline$g \rightarrow Q \bar{Q}$ & $\frac{m^{2}}{(1-z)}$ \\
\hline
\end{tabular}

Note that the $J_{P \rightarrow H}$ can also be obtained from the final state $K_{C \rightarrow A}$ by appropriate crossings.

The final state contributions to the crossing functions are given by

$$
C_{H, \text { final }}^{\mathcal{H}}(x)=\left(\frac{N}{2 \pi}\right) f_{H}^{\mathcal{H}}(x) \sum_{P} I_{H \rightarrow P U}\left(z_{1}, z_{2}\right),
$$

where the $I_{H \rightarrow P U}$ are given in Eq. (126-128). These contributions have to be subtracted:

$$
C_{H}^{\mathcal{H}}(x)=C_{H, \text { init }}^{\mathcal{H}}(x)-C_{H, \text { final }}^{\mathcal{H}}(x) .
$$

Defining the functions $X_{P \rightarrow H}(z, m)$ by

$$
C_{H}^{\mathcal{H}}(x)=\sum_{P} \int_{x}^{1} \frac{d z}{z} f_{P}^{\mathcal{H}}(x / z) X_{P \rightarrow H}(z, m),
$$

the four heavy quark contributions are given by,

$$
\begin{aligned}
X_{Q \rightarrow Q}(z, m) & =\left(\frac{N}{2 \pi}\right)\left(J_{Q \rightarrow Q}\left(z, z_{2}\right)-\delta(1-z) I_{Q \rightarrow Q g}\left(0, z_{2}\right)\right), \\
& =\left(\frac{N}{2 \pi}\right) \frac{1}{2}\left(1-\frac{1}{N^{2}}\right)\left[\left(\frac{1+z^{2}}{1-z}\right)_{+} \ln \left(\frac{s_{\min }}{m^{2}}\right)\right. \\
& \left.-\frac{2 z}{(1-z)_{+}}-\left(1+z^{2}\right)\left(\frac{\ln (1-z)}{1-z}\right)_{+}+\left(\frac{\pi^{2}}{3}-\frac{3}{2}\right) \delta(1-z)\right], \\
X_{g \rightarrow Q}(z, m) & =\left(\frac{N}{2 \pi}\right) J_{g \rightarrow Q}(z), \\
X_{Q \rightarrow g}(z, m) & =\left(\frac{N}{2 \pi}\right) J_{Q \rightarrow g}(z), \\
X_{g \rightarrow g}^{m}(z, m) & =-\frac{N}{2 \pi} I_{g \rightarrow Q \bar{Q}}(0,0) \delta(1-z) .
\end{aligned}
$$

where $X_{g \rightarrow g}^{m}$ is the massive quark contribution to the massless $X_{g \rightarrow g}$.

After factorization, using the minimal subtraction scheme defined by the massive counterterm functions given in Eq. (138-141) (and replacing $\mu_{D}$ by $\mu_{F}$ ), the crossing function can be written as in the massless case:

$$
C_{H}^{\mathcal{H}, \mathrm{MS}}\left(x, m, \mu_{F}\right)=\frac{N}{2 \pi}\left[A_{H}^{\mathcal{H}}\left(x, \mu_{F}\right) \ln \left(\frac{s_{\text {min }}}{\mu_{F}^{2}}\right)+B_{H}^{\mathcal{H}, \mathrm{MS}}\left(x, \mu_{F}\right)\right],
$$


with

$$
A_{H}^{\mathcal{H}}(x)=\sum_{P} A_{P \rightarrow H}^{\mathcal{H}}\left(x, \mu_{F}\right) \quad, \quad B_{H}^{\mathcal{H}, \mathrm{MS}}(x)=\sum_{P} B_{P \rightarrow H}^{\mathcal{H}}\left(x, \mu_{F}\right)
$$

we have

$$
\begin{gathered}
A_{Q \rightarrow Q}^{\mathcal{H}}\left(x, \mu_{F}\right)=\frac{1}{2}\left(1-\frac{1}{N^{2}}\right) \int_{x}^{1} \frac{d z}{z} f_{Q}^{\mathcal{H}}\left(x / z, \mu_{F}\right)\left[\left(\frac{1+z^{2}}{1-z}\right)_{+}\right], \\
A_{g \rightarrow Q}^{\mathcal{H}}\left(x, \mu_{F}\right)=\frac{1}{4} \int_{x}^{1} \frac{d z}{z} f_{g}^{\mathcal{H}}\left(x / z, \mu_{F}\right) P_{g \rightarrow q \bar{q}}^{\epsilon=0}(z), \\
A_{Q \rightarrow g}^{\mathcal{H}}\left(x, \mu_{F}\right)=\frac{1}{4} \int_{x}^{1} \frac{d z}{z} f_{Q}^{\mathcal{H}}\left(x / z, \mu_{F}\right) P_{q \rightarrow g q}^{\epsilon=0}(z), \\
A_{g \rightarrow g}^{m, \mathcal{H}}\left(x, \mu_{F}\right)=-\frac{1}{3 N} f_{g}^{\mathcal{H}}\left(x, \mu_{F}\right),
\end{gathered}
$$

and for the scheme dependent functions

$$
\begin{gathered}
B_{Q \rightarrow Q}^{\mathcal{H}, \mathrm{MS}}\left(x, \mu_{F}\right)=\frac{1}{2}\left(1-\frac{1}{N^{2}}\right) \int_{x}^{1} \frac{d z}{z} f_{Q}^{\mathcal{H}}\left(x / z, \mu_{F}\right)\left[\left(\frac{\pi^{2}}{3}-\frac{3}{2}\right) \delta(1-z)\right. \\
\left.-\frac{2 z}{(1-z)_{+}}-\left(1+z^{2}\right)\left(\frac{\ln (1-z)}{1-z}\right)_{+}\right], \\
B_{g \rightarrow Q}^{\mathcal{H}, \mathrm{MS}}\left(x, \mu_{F}\right)=\frac{1}{2 N} \int_{x}^{1} \frac{d z}{z} f_{g}^{\mathcal{H}}\left(x / z, \mu_{F}\right)\left[\left(z^{2}+(1-z)^{2}\right) \ln (1-z)+2 z(1-z)\right], \\
B_{Q \rightarrow g}^{\mathcal{H}, \mathrm{MS}}\left(x, \mu_{F}\right)=\frac{1}{2}\left(1-\frac{1}{N^{2}}\right) \int_{x}^{1} \frac{d z}{z} f_{Q}^{\mathcal{H}}\left(x / z, \mu_{F}\right)\left[\frac{1+(1-z)^{2}}{z} \ln \left(\frac{1-z}{z^{2}}\right)-\frac{2(1-z)}{z}\right], \\
B_{g \rightarrow g}^{m, \mathcal{H}, \mathrm{MS}}\left(x, \mu_{F}\right)=\frac{5}{9 N} f_{g}^{\mathcal{H}}\left(x, \mu_{F}\right) .
\end{gathered}
$$

Just as for the tagging functions the massive quark contributions to $A_{g \rightarrow g}$ and $B_{g \rightarrow g}$ are equivalent to a change of $n_{f}$ by one.

It is easy to verify that the choice of the massive $\overline{\mathrm{MMS}}$ counterterms in Eq. (150-153) yields crossing functions that are equal to those of the massless case (given in Eq. (3.39) and (3.40) 团) in Ref. 5.

In both factorization schemes the large logarithms $\ln (\mathcal{Q} / m)$ due to initial state collinear radiation are resummed via the evolution of the parton distribution functions.

\section{Matching at low $Q$}

The PSS method, with the extensions presented in this paper, provides a natural framework for the implementation of a variable flavor number scheme (VFNS) [26, 27, 28, 29, 30,

\footnotetext{
${ }^{4}$ We note a typo in Eq. (3.40), the sign after the delta function in the expression for $B_{q \rightarrow q}^{\mathcal{H}, \overline{\mathrm{MS}}}$ should be changed.
} 
31.32 in the calculation of differential cross sections. The name refers to the fact that in such a scheme the number of active flavors depends on the value of $Q$. Here we merely sketch the outlines of such an implementation, we hope to provide a complete example in the future [2]. For a review of recent VFNS developments see e.g. Refs. [31,36].

When the typical scale $Q$ is close to the mass of the heavy quark, we may use the PSS method extended by the soft gluon radiation results of section 4.1. There is no need to compute analytically the contribution of (approximately) collinear configurations involving the heavy quark, a numerical integration should suffice. The heavy quark is not treated as a parton, neither in the initial nor in the final state, i.e. it does not contribute to the running of $\alpha_{s}$, nor to the evolution of the parton distribution functions or fragmentation functions. To calculate the production cross section of a $D$ or $B$ meson, we have to convolute the parton-level results with a non-evolving fragmentation function (e.g. a Peterson-Zerwas [37] function, or simply $D_{H}^{\mathcal{H}}(z)=\delta(1-z)$ ). This calculational scheme, when adopted irrespective of $\mathcal{Q}$, is called a fixed flavor number scheme (FFNS).

When $Q$ is much larger than $m$, the fully extended PSS method should be used, including the heavy quark crossing and tagging functions, factorized e.g in the $\overline{\text { MMS }}$ scheme, so that large logarithms $\ln (2 / m)$ are resummed, see earlier in this section. Now the heavy quark is treated as a parton, and it contributes to the evolution of $\alpha_{s}$, the parton distribution functions and fragmentation functions.

The issue is now how we should merge these two descriptions into one calculational scheme. For the discussion here we assume that the factorization theorem holds even at a low scale close to the mass of the heavy quark, see Ref. [31. We take $\mu_{R}=\mu_{F}=\mu$ for simplicity. At the end we shall put $\mu=2$.

Let us concentrate on the initial state. We have in mind a cross section sufficiently inclusive to not require any fragmentation and tagging functions. The corresponding matrix element is computed with all partons in the final state, including the heavy quarks. Renormalization is performed in the CWZ scheme [22], and all soft and collinear singularities have cancelled due to the KLN [16] theorem.

We start by choosing a matching scale, $\mu_{0} \simeq m$, such that, for $\mu<\mu_{0}$, we use $n_{l}$ active parton flavors in our calculation and treat the heavy quark as non-partonic, while for $\mu>\mu_{0}$ we use $n_{l}+1$ active flavors by treating also the heavy quark as partonic in the sense that it participates in the evolution of $\alpha_{s}$ and the parton distribution functions.

For $\mu<\mu_{0}$ we cross, following Eq. (50), all possible $\left(n_{l}\right)$ light partons to the initial state, and fold the result of each crossing with the corresponding $n_{l}$-flavor parton distributions functions. We also add the contributions from the corresponding ( $\overline{\mathrm{MS}})$ light parton crossing functions. The evolution of the parton distribution functions involves only the light degrees of freedom.

\footnotetext{
${ }^{5}$ E.g. one might think of the deep-inelastic heavy-quark structure function.
} 
For $\mu>\mu_{0}$ we replace all $n_{l}$-flavor parton distribution functions and $\alpha_{s}$ by their $n_{l}+1$ versions. The relations between these $n_{l}$ and $n_{l}+1$ quantities are given by socalled matching conditions, computed for $\alpha_{s}$ to two-loop in Ref. 38] and for the parton distribution functions to one and two-loop in Ref. [39,27]. We extend the sums over parton flavors and their crossings in Eq. (50) to include the heavy quark. However, where we should add a heavy quark crossing function, we only need to add the corresponding counterterm function (e.g. those in Eqs. (150-153)). This is because, in the matrix element, all contributions, including near-collinear ones, are computed numerically . Of course, for $\mu \gg \mu_{0}$ one may use the heavy quark crossing functions derived earlier in this section to compute the contributions of the collinear region and counterterm functions.

To show that this calculational scheme constitutes a VFNS, we must verify that both the low and high $\mathcal{Q}$ descriptions are properly reproduced. Clearly, when $\mu \gg \mu_{0}$, we have an $n_{l}+1$ flavor description, where the large logarithm $\ln (\mathcal{Q} / m)$, converted by the counterterm functions into $\ln (\mathcal{Q} / \mu)$, may be eliminated from the matrix element and resummed into the parton distribution function by the choice $\mu=\mathcal{Q}$.

For $\mu$ just above $\mu_{0}$ the requirement is that the cross section computed in the VFNS is the same as computed in the FFNS]. The argument essentially follows Ref. [26]. For $\mu>\mu_{0}$, the difference with the FFNS cross section is mainly due to the evolution of the gluon distribution function caused by gluon splitting into a heavy quark pair, and the counterterm functions. For $\mu \gtrsim m$ (putting $\mu_{0}=m$, and suppressing all irrelevant arguments), the former contribution can be written as

$$
d \sigma_{\mathrm{NLO}}^{\left(n_{l}+1\right)}(\mathrm{VFNS})-d \sigma_{\mathrm{NLO}}^{\left(n_{l}\right)}(\mathrm{FFNS}) \simeq\left(\alpha_{s}\left(\frac{N}{2 \pi}\right) \ln \left(\frac{\mu}{m}\right) \frac{1}{2} P_{g \rightarrow q \bar{q}}\right) d \sigma_{\mathrm{LO}}^{\left(n_{l}\right)}
$$

with $\mu$ not much larger than $m$. The corresponding counterterm functions (see e.g. Eq. (140)), precisely cancels the $O\left(\alpha_{s}\right)$ term in Eq. (180), as required.

\section{Conclusions}

In this paper we extended the phase space slicing method of Giele, Glover and Kosower [4.5] for computing NLO corrections to jet cross sections to incorporate fragmentation functions and heavy quarks. This makes the method applicable to any reaction in which a particular final state hadron (light or heavy) is tagged.

\footnotetext{
${ }^{6}$ For $\mu=Q$ not too much larger than $\mu_{0}$, neglecting terms of order $m^{2} / s_{\min }$ when computing heavy quark crossing and tagging functions, is not very accurate, due to the constraint $s_{\min } \ll Q^{2}$. Here it is therefore better to compute contributions from the collinear region numerically.

${ }^{7}$ Note that we consider one loop matching here, in which $\alpha_{s}$ and the parton distribution functions are continuous across $\mu_{0}$, in the $\overline{\mathrm{MS}}$ scheme.
} 
The extension to fragmentation functions for massless partons amounted to a generalization of the crossing function approach [5] to the final state, and led us to introduce a new class of functions we named tagging functions.

The extension to heavy quarks consisted of two parts. Effects of soft radiation off heavy quarks were straightforwardly included. Contributions from collinear radiation involving heavy quarks were included in heavy quark crossing and tagging functions. We

showed that heavy quark tagging functions led naturally to the Mele-Nason perturbative fragmentation functions [25]. We described briefly how our results might be included in a variable flavor number scheme.

In this paper we developed the formalism of the extended PSS method. Its practical use, and its application in the context of a variable flavor number scheme, will be assessed in future work [2].

\section{Acknowledgments}

We would like to thank Walter Giele, Jack Smith and Wu-Ki Tung for useful discussions. E.L. would like to thank the Institute for Theoretical Physics in Stony Brook for its hospitality. Part of this work was done while S.K was at Fermilab.

\section{References}

[1] F. Abe et al (CDF collaboration), Phys. Rev. Lett. 74 (1995) 2626; S. Abachi et al (D0 collaboration), Phys. Rev. Lett. 74 (1995) 2632.

[2] S. Keller and E. Laenen, in preparation.

[3] H. Baer, J. Ohnemus, and J. F. Owens, Phys. Rev. D40 (1989) 2844; K. Fabricius, G. Kramer, G. Schierholz and I. Schmitt, Z. Phys. C11 (1981) 315; G. Kramer and B. Lampe, Fortschr. Phys. 37 (1989) 161.

[4] W.T. Giele and E.W.N. Glover, Phys. Rev. D46 (1992) 1980.

[5] W.T. Giele, E.W.N. Glover, and D.A. Kosower, Nucl. Phys. B403 (1993) 633.

[6] R.K. Ellis, D.A. Ross and A.E. Terrano, Nucl. Phys. B178, (1988) 421; Z. Kunszt, P. Nason, "Z Physics at LEP1", CERN 89-08, vol.1, p373.

[7] M.L. Mangano, P. Nason, and G. Ridolfi, Nucl. Phys. B373 (1992) 295; S. Frixione, M.L. Mangano, P. Nason and G. Ridolfi, Nucl. Phys. B403 (1993) 633; B.W. Harris and J. Smith, Nucl. Phys. B452 (1995) 109; B.W. Harris and J.F. Owens, Phys. Rev. D54 (1996) 2295. 
[8] S. Catani, M. Seymour, Nucl. Phys. B485 (1997) 291; Phys. Lett. B378 (1996) 287.

[9] S. Catani, M. Seymour, in preparation.

[10] J.A.M. Vermaseren, K.J.F. Gaemers and S.J. Oldham, Nucl. Phys. B187 (1981) 301; W.L. van Neerven and J.A.M. Vermaseren, Nucl. Phys. B238 (1984) 73.

[11] W.T. Giele, S. Keller and E. Laenen, Phys. Lett. B372 (1996) 141. M. Krämer and E. Laenen, Phys. Lett. B371 (1996) 303; U. Baur, S. Keller and D. Wackeroth, hep-ph/9611332, Proceedings of 1996 DPF / DPB Summer Study on New Directions for High-energy Physics (Snowmass 96).

[12] S. Keller and E. Laenen, hep-ph/9609383, Proceedings of 1996 DPF Meeting of the American Physical Society, Minneapolis, Aug 1996.

[13] F.A. Berends and W.T Giele, Nucl. Phys. B294 (1987) 700; M. Mangano, S. Parke and Z. Xu, Nucl. Phys. B298 (673) 653, Nucl. Phys. B299 (1988) 673; Z. Bern and D.A. Kosower, Nucl. Phys. B362 (1991) 389; D. Zeppenfeld, Int. J. Mod. Phys. A3 (1988) 2174.

[14] F.A. Berends and W.T Giele, Nucl. Phys. B313 (1989) 595.

[15] G. 't Hooft, Nucl. Phys. B33 (1971) 173; Nucl. Phys. B35 (1971) 167, G. 't Hooft and M. Veltman, Nucl. Phys. B50 (1972) 318.

[16] T. Kinoshita, J.Math.Phys. 3 (1962) 650; T.D. Lee and M. Nauenberg, Phys. Rev. 133 (1964) 1549. F. Block and A. Nordsieck, Phys. Rev. 52 (1937) 54.

[17] J.C. Collins, D.E. Soper, and G. Sterman, in "Perturbative QCD", ed. A.H. Mueller, World Scientific, Singapore 1989, p1.

[18] G. Glück, E. Reya and A. Vogt, Z. Phys. C67 (1995) 433.

[19] H.L. Lai et al, Phys. Rev. D55 (1997) 1280.

[20] A.D. Martin, R.G. Roberts, W.J. Stirling, Phys. Lett. B387 (1996) 419.

[21] J. Binnewies, B.A. Kniehl and G. Kramer, Z. Phys. C76 (1997) 677; Z. Phys. C65 (1995) 471; P. Chiapetta et al, Nucl. Phys. B412 (1994) 3; M. Greco and S. Rolli, Z. Phys. C60 (1993) 169; M. Greco, S. Rolli and A. Vicini, Z. Phys. C65 (1995) 277; P. Nason and B.R. Webber, Nucl. Phys. B421 (1994) 473.

[22] J.C. Collins, F. Wilczek, and A. Zee, Phys. Rev. D18 (1978) 242;

[23] M. Krämer and E. Laenen, in Ref 11; A. Brandenburg and P. Uwer, Nucl. Phys. 515 (1998) 279; G. Rodrigo, A. Santamaria and M. Bilenky, Phys. Rev. Lett. 79 (1997) 193. 
[24] W.T. Giele, S. Keller and E. Laenen, in Ref. 11.

[25] B. Mele and P. Nason, Nucl. Phys. B361 (1991) 626.

[26] M.A.G. Aivazis, J.C. Collins, F.I. Olness and W-K. Tung, Phys. Rev. D50 (1994) 3083, Phys. Rev. D50 (1994) 3102.

[27] M. Buza, Y. Matiounine, J. Smith, R. Migneron and W.L. van Neerven, Nucl. Phys. B472 (1996) 611; M. Buza, Y. Matiounine, J. Smith and W.L. van Neerven, Eur. Phys. J. C1 (1998) 301, Phys. Lett. B411 (1997) 211.

[28] H.L. Lai and W.K. Tung, Z. Phys. C74 (1997) 463.

[29] A.D. Martin, R.G. Roberts, M.G. Ryskin and W.J. Stirling, Eur. Phys. J. C2 (1998) 287.

[30] R.S. Thorne and R.G. Roberts, Phys. Rev. D57 (1998) 6871; Phys. Lett. B421 (1998) 303.

[31] J. C. Collins, Phys. Rev. D58 (1998) 094002.

[32] F.I. Olness, R.J. Scalise and W-K. Tung, hep-ph/9712494.

[33] G. Altarelli and G. Parisi, Nucl. Phys. B126 (1977) 298.

[34] M. Cacciari and M. Greco, Nucl. Phys. B421 (1994) 530; B. Kniehl, M. Krämer, G. Kramer and M. Spira, Phys. Lett. 356B (1995) 539; M. Cacciari and M. Greco, Z. Phys. C69 (1996) 459. M. Cacciari, M. Greco and P. Nason, J. High Energy Phys. 5 (1998) 7.

[35] P. Nason and C. Oleari, Phys. Lett. B418 (1998) 199; hep-ph/9811206.

[36] J. Smith, Proceedings of the Ringberg Workshop on New Trends in HERA Physics, Ringberg Castle, Germany, May 1997, hep-ph/9708212.

[37] C. Peterson, D. Schlatter, I. Schmitt and P. Zerwas, Phys. Rev. D27 (1983) 105.

[38] K.G. Chetyrkin, B.A. Kniehl and M. Steinhauser, Nucl. Phys. B510 (1998) 61; W. Bernreuther and W. Wetzel, Nucl. Phys. B197 (1982) 228; W. Bernreuther, Ann. Phys. (N.Y.) 151 (1983) 127.

[39] J.C. Collins and W-K. Tung Nucl. Phys. B278 (1986) 934. 